\title{
Polarization-resolved angular patterns of nematic liquid crystal cells: Topological events driven by incident light polarization
}

\author{
Alexei D. Kiselev, ${ }^{1,2, *}$ Roman G. Vovk, ${ }^{1, \dagger}$ Roman I. Egorov, ${ }^{1, \$}$ and Vladimir G. Chigrinov ${ }^{2, \S}$ \\ ${ }^{1}$ Institute of Physics of National Academy of Sciences of Ukraine, prospekt Nauki 46, 03028 Kyï, Ukraine \\ ${ }^{2}$ Hong Kong University of Science and Technology, Clear Water Bay, Kowloon, Hong Kong \\ (Received 16 May 2008; revised manuscript received 5 August 2008; published 10 September 2008)
}

\begin{abstract}
We study the angular structure of polarization of light transmitted through a nematic liquid crystal (NLC) cell by analyzing the polarization state as a function of the incidence angles and the polarization of the incident wave. The polarization-resolved angular (conoscopic) patterns emerging after the NLC cell illuminated by the convergent light beam are described in terms of the polarization singularities such as $C$ points (points of circular polarization) and $L$ lines (lines of linear polarization). For the homeotropically aligned cell, the Stokes polarimetry technique is used to measure the polarization resolved conoscopic patterns at different values of the ellipticity of the incident light, $\epsilon_{\mathrm{ell}}^{(\mathrm{inc})}$, impinging onto the cell. Using the exact analytical expressions for the transfer matrix we show that variations of the ellipticity, $\epsilon_{\mathrm{ell}}^{(\mathrm{inc})}$, induce transformations of the angular pattern exhibiting the effect of avoided L-line crossings and characterized by topological events such as creation and annihilation of the $C$ points. The predictions of the theory are found to be in good agreement with the experimental results.
\end{abstract}

DOI: 10.1103/PhysRevA.78.033815

PACS number(s): 42.25.Ja, 78.20.Fm, 42.70.Df, 42.25.Bs

\section{INTRODUCTION}

Singularities that represent structurally stable topological defects have long been known to play a pivotal role in condensed matter physics [1]. They are of particular importance in determining the properties of systems with a broken continuous symmetry [2-5] (for a recent review see, e.g., Ref. [6]). Topological methods of quantum field and gauge theories have been extensively used to classify the topological defects and to describe transformations of the singularities in ordered media such as superfluids and liquid crystals [7-9].

For electromagnetic vector fields, it was originally recognized by Nye [10-12] that the so-called polarization singularities are the important elements characterizing geometry of the Stokes parameter fields. In particular, the polarization singularities such as the $C$ points (the points where the light wave is circularly polarized) and the L lines (the curves along which the polarization is linear) frequently emerge as the characteristic feature of certain polarization state distributions. Over the past two decades these singularities and related issues have been the subject of numerous theoretical, experimental, and numerical studies [13-24].

The theory of polarization singularities has also been found to be a useful tool for studying optical properties of anisotropic media. In Ref. [25], it was applied to study the angular dependence of the polarization state of the electric displacement field for plane wave eigenmodes in birefringent dichroic chiral crystals. This analysis was then generalized and extended to a more complicated case of bianisotropic media [26].

The experimental results and theoretical analysis presented in Refs. [27,28] deal with the unfolding of a linearly

\footnotetext{
*kiselev@iop.kiev.ua

†roman.vovk@gmail.com

†rommel.ua@gmail.com

§eechigr@ust.hk
}

polarized Laguerre-Gauss $\left(\mathrm{LG}_{01}\right)$ beam with an on-axis vortex on propagation through a birefringent crystal. It was found that a complicated pattern of polarization singularities is formed as a result of the anisotropy induced symmetry breaking.

In our recent investigation into the angular distributions of the Stokes parameters describing the polarization structure behind the conoscopic images we performed a theoretical analysis of the polarization state of the light transmitted through nematic liquid crystal (NLC) cells as a function of the incidence angles $[29,30]$. This polarization structure-the so-called polarization-resolved angular (conoscopic) pattern-is represented by the field of polarization ellipses and results from the interference of four eigenmodes excited in NLC cells by the plane waves with varying direction of incidence.

NLCs are technologically important as materials where the optical anisotropy is determined by the orientational structure which is sensitive to external fields and, in restricted geometries, can also be influenced by changing the boundary conditions [31-33]. The conoscopy is widely used as an experimental technique to characterize orientational structures in NLC cells.

For example, this method was employed to detect biaxiality of NLCs $[34,35]$ and to measure the pretilt angle in uniaxial liquid crystal cells [36,37]. Orientational structures and helix unwinding process in ferroelectric and antiferroelectric smectic liquid crystals were also studied by conoscopy in Refs. [38-40].

In this paper we explore both theoretically and experimentally the polarization-resolved angular patterns for convergent light beam impinging on the homeotropically aligned NLC cell at varying polarization of the incident wave. The layout of the paper is as follows. In Sec. II we give experimental details and describe our setup employed to carry out measurements using a suitably modified method of the Stokes polarimetry $[27,41-43]$. The problem of light trans- 


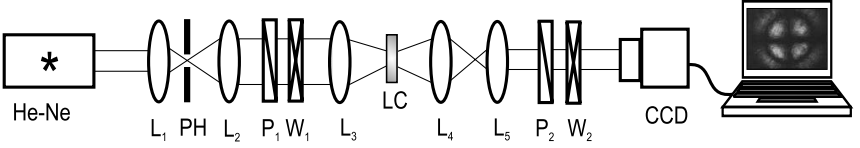

FIG. 1. Experimental setup: He-Ne is the laser; $L_{1}, L_{2}$, and $L_{5}$ are the collimating lenses; $L_{3}$ and $L_{4}$ are the microscope objectives; $\mathrm{PH}$ is the pinhole; $\mathrm{LC}$ is the NLC cell; $P_{1}$ and $P_{2}$ are the polarizers; $W_{1}$ and $W_{2}$ are the quarter wave plates; CCD is the CCD camera. A microscope objective $L_{3}$ is illuminated with an elliptically polarized and expanded parallel beam of light from a He-Ne laser. The output from a second objective $L_{4}$ is collected by a CCD camera through the Stokes analyzer.

mission through a uniformly anisotropic NLC cell is considered in Sec. III A. Using the $4 \times 4$ matrix formalism we deduce the general expressions relating the evolution matrix to the transmission and reflection matrices. The analytical results are used for analysis of the polarization-resolved conoscopic patterns in Sec. III B. The patterns emerging after homeotropically aligned NLC cells are treated in Sec. III C. The polarization ellipse fields are studied as a function of the ellipticity $\epsilon_{\mathrm{ell}}^{\text {(inc) }}$, and the polarization azimuth $\phi_{p}^{(\mathrm{inc})}$, characterizing the polarization state of the incident wave. The analytical expressions describing loci of the $C$ points and $L$ lines along with the index formula are used to examine rearrangements of the polarization singularities caused by variations of the polarization parameters $\epsilon_{\mathrm{ell}}^{\text {(inc) }}$ and $\phi_{p}^{(\mathrm{inc})}$. We find that changing the azimuth results in rotation of the ellipse field as a whole by the angle $\phi_{p}^{\text {(inc) }}$, whereas the transformations induced by the ellipticity appear to be complicated by the presence of bifurcations leading to creation and annihilation of the $C$ points. It is shown that the structure of the intersecting $L$ lines formed at $\epsilon_{\mathrm{ell}}^{(\mathrm{inc})}=0$ smoothly evolve into a family of the concentric $L$ circles at $\left|\epsilon_{\mathrm{ell}}^{(\mathrm{inc})}\right|=1$. At small values of the ellipticity, the effect of avoided $L$ line crossings is found to be an important feature of this transition. Experimentally measured and computed fields of the polarization ellipses are presented in Sec. IV along with discussion and concluding remarks. Mathematical details on the evolution operator of uniformly anisotropic media and related issues are relegated to the Appendix.

\section{EXPERIMENT}

In our experiments we used the NLC cells of thickness $d=110 \mu \mathrm{m}$ filled with the nematic liquid crystal mixture E7 from Merk. Two glass substrates were assembled to form a hometropically oriented NLC cell. At the wavelength of light generated by a low power He-Ne laser from Coherent Group with $\lambda=632.8 \mathrm{~nm}$ (see Fig. 1), the ordinary and extraordinary refractive indices of the NLC are $n_{o}=1.5246$ and $n_{e}$ $=1.7608$, respectively; the refractive index of the glass substrates is $n_{g}=1.5$.

Figure 1 shows our experimental setup devised to perform the conoscopic measurements using the Stokes polarimetry technique [27,41-43]. Referring to Fig. 1, the cell is irradiated with a convergent light beam formed by the microscope objective of high numerical aperture $L_{3}$. The input polarizer $P_{1}$ is combined with the properly oriented quarter wave plate
$W_{1}$ to control the polarization characteristics (the ellipticity and the azimuth of polarization) of a He-Ne laser beam which is expanded and collimated using the lenses $L_{1}$ and $L_{2}$. A charge coupled device (CCD) camera collects the output from the microscope objective $L_{4}$ through the collimating lens $L_{5}$ and the Stokes analyzer represented by the combination of the quarter wave plate $W_{2}$ and the polarizer $P_{2}$.

This optical arrangement therefore collects simultaneously the transmittance of the cell for a range of incident angles. The distribution of the Stokes parameters $S_{0}, \ldots, S_{3}$ describing the state of polarization of the transmitted light can then be obtained by performing the measurements at six different combinations of the quarter wave plate $W_{2}$ and the analyzer $P_{2}$ and using the well-known relations [44]

$$
\begin{gathered}
S_{1}=I_{0^{\circ}}-I_{90^{\circ}}, \\
S_{2}=I_{45^{\circ}}-I_{135^{\circ}}, \\
S_{3}=I_{\mathrm{RCP}}-I_{\mathrm{LCP}}, \\
S_{0}=\sqrt{S_{1}^{2}+S_{2}^{2}+S_{3}^{2}},
\end{gathered}
$$

where $I_{0^{\circ}}, I_{90^{\circ}}, I_{45^{\circ}}$, and $I_{135^{\circ}}$ are the linearly polarized components (the subscript indicates the orientation angle of the analyzer); $I_{\mathrm{RCP}}$ and $I_{\mathrm{LCP}}$ are the right- and left-handed circular polarized components measured in the presence of the quarter wave plate $W_{2}$.

Geometrically, the distribution of the Stokes parameters measured in the observation plane can be conveniently represented by the two-dimensional field of the polarization ellipses. The geometrical elements of polarization ellipses that determine the polarization ellipse field can be readily computed from the Stokes parameters (1).

The orientation of a polarization ellipse is specified by the azimuthal angle of polarization (polarization azimuth)

$$
\phi_{p}=\frac{1}{2} \arg \left(S_{1}+i S_{2}\right),
$$

and its eccentricity is described by the signed ellipticity parameter

$$
\epsilon_{\mathrm{ell}}=\tan \left[\frac{1}{2} \arcsin \left(\frac{S_{3}}{S_{0}}\right)\right] .
$$

This parameter will be referred to as the ellipticity. The handedness of the ellipse is determined by the sign of the ellipticity parameter $\epsilon_{\text {ell }}$.

In Sec. IV, we shall describe the polarization ellipse fields representing the experimentally measured angular patterns. These will be compared with the predictions of the theory discussed in the subsequent section.

\section{THEORY}

\section{A. Transmission boundary-value problem}

We consider a nematic liquid crystal (NLC) cell of thickness $d$ sandwiched between two parallel plates that are normal to the $z$ axis: $z=0$ and $z=d$. Typically, anisotropy of 
nematics is locally uniaxial and NLC molecules align on average along a local unit director $\hat{\mathbf{d}}$ [31]. In this case the NLC director

$$
\hat{\mathbf{d}} \equiv \mathbf{e}_{0}(\hat{\mathbf{d}})=\sin \theta_{d} \cos \phi_{d} \hat{\mathbf{x}}+\sin \theta_{d} \sin \phi_{d} \hat{\mathbf{y}}+\cos \theta_{d} \hat{\mathbf{z}}
$$

determines the optic axis. So, the uniaxially anisotropic dielectric tensor $\varepsilon$ is given by

$$
\varepsilon=\epsilon_{\perp} \mathbf{I}_{3}+\Delta \epsilon \hat{\mathbf{d}} \otimes \hat{\mathbf{d}}, \quad \Delta \epsilon=\epsilon_{\|}-\epsilon_{\perp},
$$

where $\mathbf{I}_{n}$ is the $n \times n$ identity matrix. (In what follows carets will denote unit vectors.) Its two principal values $\epsilon_{\perp}$ and $\epsilon_{\|}$ define the ordinary and extraordinary refractive indices, $n_{o}$ $=\sqrt{\mu \epsilon_{\perp}}$ and $n_{e}=\sqrt{\mu \epsilon_{\|}}$, where $\mu$ is the NLC magnetic permeability.

In a more general case of biaxial nematics $[45,46]$ that were recently observed experimentally [35,47], there are three different dielectric constants $\epsilon_{1}, \epsilon_{2}$, and $\epsilon_{3}$ representing the eigenvalues of the dielectric tensor as follows:

$$
\boldsymbol{\varepsilon}=\epsilon_{3} \mathbf{I}_{3}+\Delta \epsilon_{1} \hat{\mathbf{d}} \otimes \hat{\mathbf{d}}+\Delta \epsilon_{2} \hat{\mathbf{m}} \otimes \hat{\mathbf{m}},
$$

where $\Delta \epsilon_{i}=\epsilon_{i}-\epsilon_{3}$, and the eigenvectors $\hat{\mathbf{d}}, \hat{\mathbf{m}}$, and $\hat{\mathbf{l}}=\hat{\mathbf{d}} \times \hat{\mathbf{m}}$ give the corresponding principal axes.

Similar to the director (4), the unit vectors $\hat{\mathbf{m}}$ and $\hat{\mathbf{I}}$ can be expressed in terms of Euler angles as follows:

$$
\begin{gathered}
\hat{\mathbf{m}}=\cos \gamma_{d} \mathbf{e}_{1}(\hat{\mathbf{d}})+\sin \gamma_{d} \mathbf{e}_{2}(\hat{\mathbf{d}}), \\
\hat{\mathbf{l}}=-\sin \gamma_{d} \mathbf{e}_{1}(\hat{\mathbf{d}})+\cos \gamma_{d} \mathbf{e}_{2}(\hat{\mathbf{d}}),
\end{gathered}
$$

where $\quad \mathbf{e}_{1}(\hat{\mathbf{d}})=\left(\cos \theta_{d} \cos \phi_{d}, \cos \theta_{d} \sin \phi_{d},-\sin \theta_{d}\right) \quad$ and $\mathbf{e}_{2}(\hat{\mathbf{d}})=\left(-\sin \phi_{d}, \cos \phi_{d}, 0\right)$.

We shall need to write the Maxwell equations for a harmonic electromagnetic wave (the time-dependent factor is $\exp \{-i \omega t\})$ in the form

$$
\begin{aligned}
& \boldsymbol{\nabla} \times \mathbf{E}=i \mu k_{\mathrm{vac}} \mathbf{H}, \\
& \boldsymbol{\nabla} \times \mathbf{H}=-i k_{\mathrm{vac}} \mathbf{D},
\end{aligned}
$$

where $k_{\mathrm{vac}}=\omega / c$ is the free-space wave number; $\mu$ is the magnetic permittivity and $\mathbf{D}=\boldsymbol{\varepsilon} \cdot \mathbf{E}$ is the electric displacement field.

The medium surrounding the NLC cell is assumed to be optically isotropic and characterized by the dielectric constant $\epsilon_{m}$ and the magnetic permittivity $\mu_{m}$. So, Maxwell's equations in the medium outside the cell can be obtained from Eq. (8) by replacing $\mu$ and $\mathbf{D}$ with $\mu_{m}$ and $\epsilon_{m} \mathbf{E}$, respectively.

As is shown in Fig. 2, there are two plane waves in the half space $z \leqslant 0$ bounded by the entrance face of the NLC cell: the incoming incident wave $\left\{\mathbf{E}_{\mathrm{inc}}, \mathbf{H}_{\mathrm{inc}}\right\}$ and the outgoing reflected wave $\left\{\mathbf{E}_{\mathrm{refl}}, \mathbf{H}_{\mathrm{refl}}\right\}$. In the half space $z \geqslant d$ after the exit face, the only wave is the transmitted plane wave $\left\{\mathbf{E}_{\mathrm{tr}}, \mathbf{H}_{\mathrm{tr}}\right\}$ which propagates along the direction of incidence and is excited by the incident light.

So, the electric field outside the cell is a superposition of the plane wave solutions of the Maxwell equations

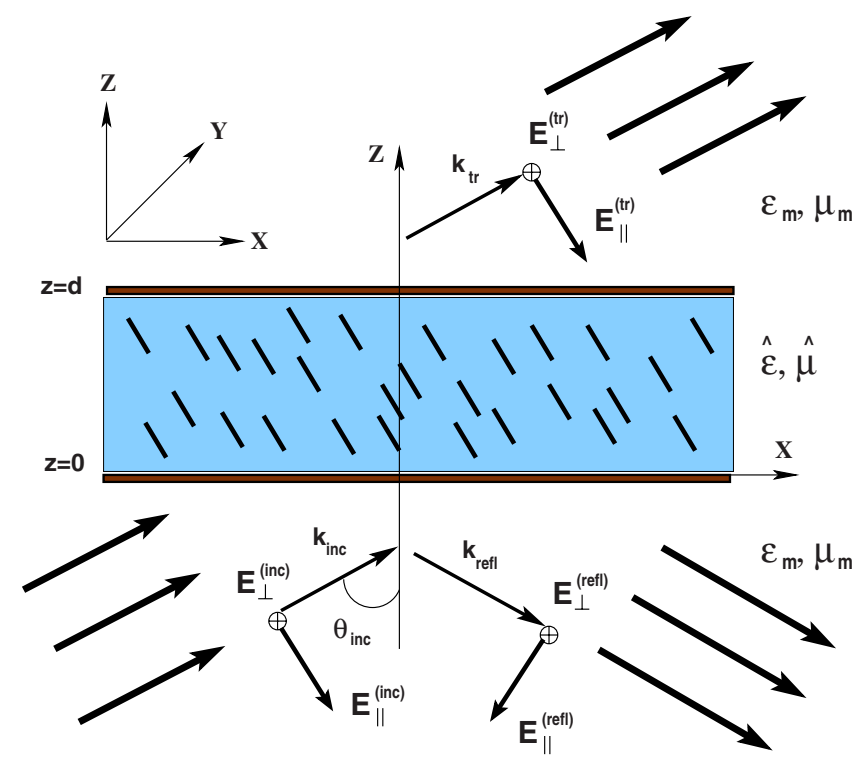

FIG. 2. (Color online) Geometry of nematic cell in the plane of incidence.

$$
\begin{gathered}
\left.\mathbf{E}\right|_{z<0}=\mathbf{E}_{\mathrm{inc}}\left(\hat{\mathbf{k}}_{\mathrm{inc}}\right) e^{i\left(\mathbf{k}_{\mathrm{inc}} \cdot \mathbf{r}\right)}+\mathbf{E}_{\mathrm{refl}}\left(\hat{\mathbf{k}}_{\mathrm{refl}}\right) e^{i\left(\mathbf{k}_{\mathrm{refl}} \cdot \mathbf{r}\right)}, \\
\left.\mathbf{E}\right|_{z>d}=\mathbf{E}_{\mathrm{tr}}\left(\hat{\mathbf{k}}_{\mathrm{tr}}\right) e^{i\left(\mathbf{k}_{\mathrm{tr}} \cdot \mathbf{r}\right)},
\end{gathered}
$$

where the wave vectors $\mathbf{k}_{\text {inc }}, \mathbf{k}_{\text {refl }}$, and $\mathbf{k}_{\mathrm{tr}}$ that are constrained to lie in the plane of incidence due to the boundary conditions requiring the tangential components of the electric and magnetic fields to be continuous at the boundary surfaces. These conditions are given by

$$
\mathbf{P}(\hat{\mathbf{z}}) \cdot\left[\left.\mathbf{E}\right|_{z=0+0}-\left.\mathbf{E}\right|_{z=0-0}\right]=\mathbf{P}(\hat{\mathbf{z}}) \cdot\left[\left.\mathbf{E}\right|_{z=d+0}-\left.\mathbf{E}\right|_{z=d-0}\right]=0,
$$

$$
\mathbf{P}(\hat{\mathbf{z}}) \cdot\left[\left.\mathbf{H}\right|_{z=0+0}-\left.\mathbf{H}\right|_{z=0-0}\right]=\mathbf{P}(\hat{\mathbf{z}}) \cdot\left[\left.\mathbf{H}\right|_{z=d+0}-\left.\mathbf{H}\right|_{z=d-0}\right]=0,
$$

where $\mathbf{P}(\hat{\mathbf{z}})=\mathbf{I}_{3}-\hat{\mathbf{z}} \otimes \hat{\mathbf{z}}$ is the projector onto the plane with the normal directed along the vector $\hat{\mathbf{z}}$ (the $x-y$ plane).

Another consequence of the boundary conditions (10) is that the tangential components of the wave vectors are the same. Assuming that the incidence plane is the $x-z$ plane we have

$$
\mathbf{k}_{\alpha}=k_{\mathrm{vac}} \mathbf{q}_{\alpha}=k_{m} \hat{\mathbf{k}}_{\alpha}=k_{x} \hat{\mathbf{x}}+k_{z}^{(\alpha)} \hat{\mathbf{z}}, \quad \alpha \in\{\text { inc, refl, } \operatorname{tr}\},
$$

where $k_{m} / k_{\mathrm{vac}}=n_{m}=\sqrt{\mu_{m} \epsilon_{m}}$ is the refractive index of the ambient medium and the components can be expressed in terms of the incidence angle $\theta_{\text {inc }}$ as follows:

$$
\begin{gathered}
k_{x}=k_{m} \sin \theta_{\mathrm{inc}} \equiv k_{\mathrm{vac}} q_{x}, \\
k_{z}^{(\mathrm{inc})}=k_{z}^{(\mathrm{tr})}=-k_{z}^{(\mathrm{refl})}=k_{m} \cos \theta_{\mathrm{inc}} \equiv k_{\mathrm{vac}} q_{z}^{(m)} .
\end{gathered}
$$

The plane wave traveling in the isotropic ambient medium along the wave vector (11) is transverse, so that the polarization vector is given by 


$$
\begin{gathered}
\mathbf{E}_{\alpha}\left(\hat{\mathbf{k}}_{\alpha}\right)=E_{\|}^{(\alpha)} \mathbf{e}_{1}\left(\hat{\mathbf{k}}_{\alpha}\right)+E_{\perp}^{(\alpha)} \mathbf{e}_{2}\left(\hat{\mathbf{k}}_{\alpha}\right), \\
\mathbf{e}_{1}\left(\hat{\mathbf{k}}_{\alpha}\right)=k_{m}^{-1}\left(k_{z}^{(\alpha)} \hat{\mathbf{x}}-k_{x} \hat{\mathbf{z}}\right), \quad \mathbf{e}_{2}\left(\hat{\mathbf{k}}_{\alpha}\right)=\hat{\mathbf{y}},
\end{gathered}
$$

where $E_{\|}^{(\alpha)}$ and $E_{\perp}^{(\alpha)}$ are the in-plane and out-of-plane components of the electric field, respectively. The vector characterizing the magnetic field is

$$
\mu_{m} \mathbf{H}_{\alpha}\left(\hat{\mathbf{k}}_{\alpha}\right)=\mathbf{q}_{\alpha} \times \mathbf{E}_{\alpha}\left(\hat{\mathbf{k}}_{\alpha}\right)=n_{m}\left[E_{\|}^{(\alpha)} \hat{\mathbf{y}}-E_{\perp}^{(\alpha)} \mathbf{e}_{1}\left(\hat{\mathbf{k}}_{\alpha}\right)\right],
$$

where $\mathbf{q}_{\alpha}=k_{\mathrm{vac}}^{-1} \mathbf{k}_{\alpha}=n_{m} \hat{\mathbf{k}}_{\alpha}$. Note that, for plane waves, the dimensionless vector

$$
\mathbf{q}=k_{\mathrm{vac}}^{-1} \mathbf{k}
$$

is parallel to $\mathbf{k}$ and its length gives the refractive index. For convenience, we shall often use this vector in place of the wave vector.

The expressions (9)-(16) give the electromagnetic field of incident, transmitted, and reflected waves propagating in the ambient medium. This field is of the general form

$$
\begin{gathered}
\{\mathbf{E}(\mathbf{r}), \mathbf{H}(\mathbf{r})\}=\{\mathbf{E}(z), \mathbf{H}(z)\} \exp \left[i\left(\mathbf{k}_{P}, \mathbf{r}\right)\right], \quad \mathbf{k}_{P}=\mathbf{P}(\hat{\mathbf{z}}) \cdot \mathbf{k}, \\
\mathbf{q}_{P}=k_{\mathrm{vac}}^{-1} \mathbf{k}_{P}=q_{P}\left[\cos \left(\phi_{\text {inc }}\right) \hat{\mathbf{x}}+\sin \left(\phi_{\text {inc }}\right) \hat{\mathbf{y}}\right],
\end{gathered}
$$

where the azimuthal angle $\phi_{\text {inc }}$ specifies orientation of the incidence plane. So, in our case, we have $\phi_{\text {inc }}=0$ and $q_{P}$ $=q_{x}$.

The representation (18) can be applied to describe the field inside the cell when the dielectric tensor is independent of the in-plane coordinates $x$ and $y$. In this case the lateral components of the electric and magnetic fields

$$
\begin{aligned}
\mathbf{E}_{P} & =\mathbf{P}(\hat{\mathbf{z}}) \cdot \mathbf{E}=E_{x} \hat{\mathbf{x}}+E_{y} \hat{\mathbf{y}} \equiv\left(\begin{array}{c}
E_{x} \\
E_{y}
\end{array}\right), \\
\mathbf{H}_{P} & =\mathbf{H} \times \hat{\mathbf{z}}=H_{y} \hat{\mathbf{x}}-H_{x} \hat{\mathbf{y}} \equiv\left(\begin{array}{c}
H_{y} \\
-H_{x}
\end{array}\right),
\end{aligned}
$$

can be conveniently combined into the vector

$$
\mathbf{F}(z) \equiv\left(\begin{array}{c}
\mathbf{E}_{P}(z) \\
\mathbf{H}_{P}(z)
\end{array}\right)=\left(\begin{array}{c}
E_{x}(z) \\
E_{y}(z) \\
H_{y}(z) \\
-H_{x}(z)
\end{array}\right) .
$$

From Maxwell's equations (8), the components normal to the substrates, $E_{z}$ and $H_{z}$, can be readily expressed in terms of the lateral components

$$
\mu H_{z}=\left(\mathbf{q}_{P}, \mathbf{E}_{P} \times \hat{\mathbf{z}}\right), \quad \epsilon_{z z} E_{z}=-\left(\hat{\mathbf{z}}, \varepsilon \cdot \mathbf{E}_{P}\right)-\left(\mathbf{q}_{P}, \mathbf{H}_{P}\right) .
$$

By using the relations (23) to eliminate the normal components we obtain the equation for the field vector (22) in the following matrix form:

$$
-i \partial_{\tau} \mathbf{F}=\mathbf{M} \cdot \mathbf{F} \equiv\left(\begin{array}{ll}
\mathbf{M}_{11} & \mathbf{M}_{12} \\
\mathbf{M}_{21} & \mathbf{M}_{22}
\end{array}\right) \cdot\left(\begin{array}{c}
\mathbf{E}_{P} \\
\mathbf{H}_{P}
\end{array}\right), \quad \tau \equiv k_{\mathrm{vac}} z,
$$

where

$$
\begin{gathered}
M_{\alpha \beta}^{(11)}=-\epsilon_{z z}^{-1} q_{\alpha}^{(P)} \epsilon_{z \beta}, \\
M_{\alpha \beta}^{(22)}=-\epsilon_{z z}^{-1} \epsilon_{\alpha z} q_{\beta}^{(P)}, \\
M_{\alpha \beta}^{(12)}=\mu \delta_{\alpha \beta}-\epsilon_{z z}^{-1} q_{\alpha}^{(P)} q_{\beta}^{(P)}, \\
M_{\alpha \beta}^{(21)}=\epsilon_{\alpha \beta}-\mu^{-1} p_{\alpha}^{(P)} p_{\beta}^{(P)}-\epsilon_{z z}^{-1} \epsilon_{\alpha z} \epsilon_{z \beta},
\end{gathered}
$$

where $\delta_{\alpha \beta}$ is the Kroneker symbol, $\mathbf{q}_{P}=k_{\mathrm{vac}}^{-1} \mathbf{k}_{P}$ and $\mathbf{p}_{P}=\hat{\mathbf{z}}$ $\times \mathbf{q}_{P}$.

The field vector (22) can now be written in the form of a general solution to the linear problem (24)

$$
\mathbf{F}(\tau)=\mathbf{U}(\tau) \cdot \mathbf{F}(0),
$$

where $\mathbf{U}(\tau)$ is the evolution operator that can be determined by solving the initial value problem

$$
-i \partial_{\tau} \mathbf{U}=\mathbf{M} \cdot \mathbf{U}, \quad \mathbf{U}(0)=\mathbf{I}_{4} .
$$

Our above notations can be regarded as a version of the well-known matrix formalism due to Berreman [48]. By using these notations we derive the boundary conditions (10) in the matrix form

$$
\begin{gathered}
\mathbf{F}_{<}=\mathbf{V}_{m} \cdot\left(\begin{array}{c}
\mathbf{E}_{\mathrm{inc}} \\
\mathbf{E}_{\mathrm{refl}}
\end{array}\right)=\mathbf{F}(0), \\
\mathbf{F}_{>}=\mathbf{V}_{m} \cdot\left(\begin{array}{c}
\mathbf{E}_{\mathrm{tr}} \\
\mathbf{0}
\end{array}\right)=\mathbf{F}(h), \quad h \equiv k_{\mathrm{vac}} d,
\end{gathered}
$$

where $\mathbf{E}_{\alpha} \equiv\left(\begin{array}{c}E_{1}^{(\alpha)} \\ \left.E_{\perp}^{(\alpha)}\right)\end{array}\right)$.

The matrix $\mathbf{V}_{m}$ relates the field vectors $\mathbf{F}_{<} \equiv \mathbf{F}(0-0)$ and $\mathbf{F}_{>} \equiv \mathbf{F}(h+0)$, and the vector amplitudes $\mathbf{E}_{\alpha}$ of the waves in the surrounding medium. From Eqs. (14)-(16), its block structure

$$
\mathbf{V}_{m}=\left(\begin{array}{cc}
\mathbf{E}_{m} & -\boldsymbol{\sigma}_{3} \mathbf{E}_{m} \\
\mathbf{H}_{m} & \boldsymbol{\sigma}_{3} \mathbf{H}_{m}
\end{array}\right)
$$

is characterized by the two diagonal $2 \times 2$ matrices

$$
\mathbf{E}_{m}=\operatorname{diag}\left(q_{z}^{(m)} / n_{m}, 1\right), \quad \mu_{m} \mathbf{H}_{m}=\operatorname{diag}\left(n_{m}, q_{z}^{(m)}\right),
$$

where $q_{z}^{(m)}=\sqrt{n_{m}^{2}-q_{x}^{2}}$ and $\boldsymbol{\sigma}_{3}=\operatorname{diag}(1,-1)$.

It is rather straightforward to check the validity of the algebraic identity for the matrix (29)

$$
\left[\mathbf{V}_{m}\right]^{T} \cdot \mathbf{G} \cdot \mathbf{V}_{m}=\mathbf{N}_{m}=N_{m} \operatorname{diag}\left(\mathbf{I}_{2},-\mathbf{I}_{2}\right), \quad \mathbf{G} \equiv\left(\begin{array}{cc}
\mathbf{0} & \mathbf{I}_{2} \\
\mathbf{I}_{2} & \mathbf{0}
\end{array}\right)
$$

where $N_{m}=2 q_{z}^{(m)} / \mu_{m}$ and the superscript $T$ indicates matrix transposition.

According to Ref. [49], identities of the form (31) can be derived as the orthogonality relations resulted from the con- 
servation law for the energy flux in nonabsorbing media. Algebraically, Eq. (31) can be used to simplify inversion of the matrix $\mathbf{V}_{m}$,

$$
\mathbf{V}_{m}^{-1}=\mathbf{N}_{m}^{-1} \cdot\left[\mathbf{V}_{m}\right]^{T} \cdot \mathbf{G},
$$

and to ease qualitative analysis (see, e.g., Ref. [50] for a more extended discussion of applications).

After substituting Eq. (26) into the boundary conditions (28a) we have

$$
\begin{gathered}
\left(\begin{array}{c}
\mathbf{E}_{\mathrm{inc}} \\
\mathbf{E}_{\mathrm{refl}}
\end{array}\right)=\mathbf{W} \cdot\left(\begin{array}{c}
\mathbf{E}_{\mathrm{tr}} \\
\mathbf{0}
\end{array}\right), \\
\mathbf{W}=\mathbf{V}_{m}^{-1} \cdot \mathbf{U}^{-1}(h) \cdot \mathbf{V}_{m}=\left(\begin{array}{ll}
\mathbf{W}_{11} & \mathbf{W}_{12} \\
\mathbf{W}_{21} & \mathbf{W}_{22}
\end{array}\right),
\end{gathered}
$$

where $\mathbf{U}^{-1} \equiv \mathbf{U}^{-1}(h)$.

From Eq. (33) we have

$$
\begin{aligned}
\left(\begin{array}{l}
E_{\|}^{(\mathrm{tr})} \\
E_{\perp}^{(\mathrm{tr})}
\end{array}\right) & =\mathbf{T} \cdot\left(\begin{array}{l}
E_{\|}^{(\mathrm{inc})} \\
E_{\perp}^{(\mathrm{inc})}
\end{array}\right), \\
\mathbf{T} & =\mathbf{W}_{11}^{-1},
\end{aligned}
$$

where $\mathbf{T}$ is the transmission (transfer) matrix linking the transmitted and incident waves.

A similar result for the reflected wave is

$$
\begin{gathered}
\left(\begin{array}{l}
E_{\|}^{(\mathrm{refl})} \\
E_{\perp}^{(\mathrm{refl})}
\end{array}\right)=\mathbf{R} \cdot\left(\begin{array}{l}
E_{\|}^{(\mathrm{inc})} \\
E_{\perp}^{(\mathrm{inc})}
\end{array}\right), \\
\mathbf{R}=\mathbf{W}_{21} \cdot \mathbf{W}_{11}^{-1}=\mathbf{W}_{21} \cdot \mathbf{T},
\end{gathered}
$$

where $\mathbf{R}$ is the reflection matrix.

For uniformly anisotropic media, the elements of the matrix (25) are constants and solving Eq. (27) gives the formula for the evolution matrix,

$$
\begin{gathered}
\mathbf{U}(\tau)=\exp \{i \mathbf{M} \tau\}=\mathbf{V} \cdot \exp \{i \boldsymbol{\Lambda} \tau\} \cdot \mathbf{V}^{-1}, \\
\boldsymbol{\Lambda}=\operatorname{diag}\left(\lambda_{1}, \lambda_{2}, \lambda_{3}, \lambda_{4}\right), \quad \mathbf{M} \cdot \mathbf{V}=\mathbf{V} \cdot \boldsymbol{\Lambda},
\end{gathered}
$$

where $\lambda_{i}$ is the eigenvalue of the matrix $\mathbf{M}$ and $\mathbf{V}$ $=\left(V_{1} V_{2} V_{3} V_{4}\right)$ is the matrix composed of the right eigenvectors, $\mathbf{M} \cdot \mathbf{V}_{i}=\lambda_{i} \mathbf{V}_{i}$. Technical details on computing the evolution operator for both biaxial and uniaxial anisotropies can be found in the Appendix. In this section we restrict ourselves to the simplest (and important for our purposes) case that occurs when the NLC cell is homeotropically aligned.

For the homeotropic director structure with $\hat{\mathbf{d}}=\hat{\mathbf{z}}$, the eigenvalue problem for the matrix $\mathbf{M}$ is easy to solve. The result is

$$
\boldsymbol{\Lambda}=\operatorname{diag}\left(q_{z}^{(e)}, q_{z}^{(o)},-q_{z}^{(e)},-q_{z}^{(o)}\right), \quad \mathbf{V}=\left(\begin{array}{cc}
\mathbf{E}_{+} & -\boldsymbol{\sigma}_{3} \mathbf{E}_{+} \\
\mathbf{H}_{+} & \boldsymbol{\sigma}_{3} \mathbf{H}_{+}
\end{array}\right),
$$

$$
\mathbf{E}_{+}=\operatorname{diag}\left(q_{z}^{(e)} / n_{\perp}, 1\right), \quad \mu \mathbf{H}_{+}=\operatorname{diag}\left(n_{\perp}, q_{z}^{(o)}\right),
$$

The analytical relations (41) can now be substituted into the expression (39) to yield the evolution matrix $\mathbf{U}^{-1}(h)$ $=\mathbf{U}(-h)$ that enter the linking matrix $\mathbf{W}$ defined in Eq. (34). The final step involves substituting the block $2 \times 2$ matrices $\mathbf{W}_{11}$ and $\mathbf{W}_{21}$ into Eqs. (36) and (38) to find the transfer and the reflection matrices in the following form:

$$
\begin{gathered}
\mathbf{T}=\operatorname{diag}\left(t_{e}, t_{o}\right)=\left[\cos \left(\boldsymbol{\Lambda}_{+} h\right)-i \boldsymbol{\Gamma}_{+} \cdot \sin \left(\boldsymbol{\Lambda}_{+} h\right)\right]^{-1}, \\
\mathbf{R}=\operatorname{diag}\left(r_{e}, r_{o}\right)=-i \boldsymbol{\sigma}_{3} \cdot \boldsymbol{\Gamma}_{-} \cdot \sin \left(\boldsymbol{\Lambda}_{+} h\right) \cdot \mathbf{T},
\end{gathered}
$$

where

$$
\begin{gathered}
\boldsymbol{\Lambda}_{+} h=\operatorname{diag}\left(q_{z}^{(e)}, q_{z}^{(o)}\right) h \equiv \operatorname{diag}\left(\delta_{e}, \delta_{o}\right), \\
\boldsymbol{\Gamma}_{ \pm}=\left(\boldsymbol{\Gamma} \pm \boldsymbol{\Gamma}^{-1}\right) / 2,
\end{gathered}
$$

$$
\boldsymbol{\Gamma}=\frac{\mu_{m}}{\mu}\left(\begin{array}{cc}
\frac{n_{o}^{2} q_{z}^{(m)}}{n_{m}^{2} q_{z}^{(e)}} & 0 \\
0 & \frac{q_{z}^{(o)}}{q_{z}^{(m)}}
\end{array}\right) \equiv \operatorname{diag}\left(\gamma_{e}, \gamma_{o}\right) \text {. }
$$

\section{B. Polarization-resolved angular patterns}

We will now proceed to a study of how the polarization properties of the transmitted wave depend on the direction of the incident beam. This direction is specified by two angles: the incidence angle $\theta_{\text {inc }}$ and the azimuthal angle of the plane of incidence $\phi_{\text {inc. }}$.

In the previous section and in the Appendix, the transmission problem was analyzed in the plane of incidence where $\phi_{\text {inc }}=0$. Clearly, when $\phi_{\text {inc }} \neq 0$, in order to have the Euler angles describing orientation of the director (4) with respect to the incidence plane we need to replace the director azimuthal angle $\phi_{d}$ with $\phi_{d}-\phi_{\text {inc }}$.

In this section dependence of the polarization parameters of transmitted waves on the angles $\theta_{\text {inc }}$ and $\phi_{\text {inc }}$ will be our primary concern. For this purpose, we assume that the transmission matrix $\mathbf{T}\left(\theta_{\text {inc }}, \theta_{d}, \phi_{d}\right)$ considered in Sec. III A and in the Appendix is changed to $\mathbf{T}\left(\theta_{\text {inc }}, \theta_{d}, \phi_{d}-\phi_{\text {inc }}\right)$.

As previously discussed in Sec. II, experimentally, the characteristics of the polarization ellipse can be determined by measuring the Stokes parameters (1). These parameters are related to the coherence matrix with the elements $\mathbf{M}_{\alpha \beta}$ $=E_{\alpha} E_{\beta}{ }^{*}$, where $\alpha, \beta \in\{\|, \perp\}[51,52]$. In the circular basis $\sqrt{2} \mathbf{e}_{ \pm}(\hat{\mathbf{k}})=\mathbf{e}_{1}(\hat{\mathbf{k}}) \pm i \mathbf{e}_{2}(\hat{\mathbf{k}})$, this matrix written as a linear combination of the Pauli matrices gives the Stokes parameters $S_{i}$, as its coefficients

$$
\mathbf{M}_{c}=\mathbf{C} \cdot \mathbf{M} \cdot \mathbf{C}^{+}=\left(\begin{array}{ll}
\left|E_{+}\right|^{2} & E_{+} E_{-}^{*} \\
E_{-} E_{+}^{*} & \left|E_{-}\right|^{2}
\end{array}\right)=2^{-1} \sum_{i=0}^{4} S_{i} \boldsymbol{\sigma}_{i},
$$

where

$$
\sigma_{0}=\mathbf{I}_{2}, \quad \boldsymbol{\sigma}_{1}=\left(\begin{array}{ll}
0 & 1 \\
1 & 0
\end{array}\right), \quad \boldsymbol{\sigma}_{2}=\left(\begin{array}{cc}
0 & -i \\
i & 0
\end{array}\right), \quad \boldsymbol{\sigma}_{3}=\left(\begin{array}{cc}
1 & 0 \\
0 & -1
\end{array}\right),
$$




$$
\left(\begin{array}{l}
E_{+} \\
E_{-}
\end{array}\right)=\mathbf{C} \cdot\left(\begin{array}{c}
E_{\|} \\
E_{\perp}
\end{array}\right), \quad \mathbf{C}=2^{-1 / 2}\left(\begin{array}{cc}
1 & -i \\
1 & i
\end{array}\right) .
$$

Since the determinant of the coherence matrix vanishes, $\operatorname{det} \mathbf{M}=0$, the Stokes parameters lie on the four-dimensional cone $S_{0}^{2}=\sum_{i=1}^{3} S_{i}^{2}$, and can be parametrized as follows:

$$
\begin{gathered}
S_{0}=\left|E_{+}\right|^{2}+\left|E_{-}\right|^{2}=\left|E_{\|}\right|^{2}+\left|E_{\perp}\right|^{2} \\
S_{1}=2 \operatorname{Re} E_{+}^{*} E_{-}=\left|E_{\|}\right|^{2}-\left|E_{\perp}\right|^{2}=S_{0} \cos 2 \chi_{p} \cos 2 \phi_{p} \\
S_{2}=2 \operatorname{Im} E_{+}^{*} E_{-}=2 \operatorname{Re} E_{\perp} E_{\|}^{*}=S_{0} \cos 2 \chi_{p} \sin 2 \phi_{p} \\
S_{3}=\left|E_{+}\right|^{2}-\left|E_{-}\right|^{2}=2 \operatorname{Im} E_{\perp} E_{\|}^{*}=S_{0} \sin 2 \chi_{p}
\end{gathered}
$$

where $0<\phi_{p} \leqslant \pi$ is the polarization azimuth (2) and $-\pi / 4$ $\leqslant \chi_{p} \leqslant \pi / 4$ is the ellipticity angle. Then, the relations expressing the ellipse characteristics in terms of the Stokes parameters are

$$
\begin{gathered}
\phi_{p}=2^{-1} \arg \left(E_{+}^{*} E_{-}\right)=2^{-1} \arg S, \quad S \equiv S_{1}+i S_{2}, \\
\epsilon_{\mathrm{ell}}=\frac{\left|E_{+}\right|-\left|E_{-}\right|}{\left|E_{-}\right|+\left|E_{+}\right|}=\tan \chi_{p}, \quad \chi_{p}=2^{-1} \arcsin \left(S_{3} / S_{0}\right) .
\end{gathered}
$$

Similar to Refs. [24,53], we have used Eqs. (47) and (50d) to define the Stokes parameter $S_{3}$ which is opposite in sign to that given in the book [44]. The ellipse is considered to be right handed $(\mathrm{RH})$ if its helicity is positive, so that $\left|E_{+}\right|$ $>\left|E_{-}\right|$and $\epsilon_{\text {ell }}>0$. In the opposite case with $\epsilon_{\text {ell }}<0$, the ellipse is left handed (LH).

In the spherical basis $\left\{\mathbf{e}_{+}, \mathbf{e}_{-}\right\}$, similar to Eq. (35), the transmission matrix

$$
\mathbf{T}_{c}=\left(\begin{array}{ll}
t_{++} & t_{+-} \\
t_{-+} & t_{--}
\end{array}\right)=\mathbf{C} \cdot \mathbf{T} \cdot \mathbf{C}^{+}
$$

relates the circular components of the incident and transmitted waves

$$
\left(\begin{array}{c}
E_{+}^{(\mathrm{rr})} \\
E_{-}^{(\mathrm{tr})}
\end{array}\right)=\mathbf{T}_{c} \cdot\left(\begin{array}{c}
E_{+}^{(\mathrm{inc})} \\
E_{-}^{(\mathrm{inc})}
\end{array}\right)
$$

where $E_{ \pm}^{(\alpha)}=\left(E_{\|}^{(\alpha)} \mp i E_{\perp}^{(\alpha)}\right) / \sqrt{2}$. So, for the transfer matrix describing the conoscopic patterns on the transverse plane of projection, we have $[29,30]$

$$
\begin{gathered}
\widetilde{\mathbf{T}}(\rho, \phi)=\exp \left(-i \phi \boldsymbol{\sigma}_{3}\right) \cdot \mathbf{T}_{c}(\rho, \phi) \cdot \exp \left(i \phi \boldsymbol{\sigma}_{3}\right), \\
\rho=r \tan \theta_{\text {inc }}, \quad \phi=\phi_{\text {inc }},
\end{gathered}
$$

where $\rho$ and $\phi$ are the polar coordinates in the observation plane $(x=\rho \cos \phi$ and $y=\rho \sin \phi$ are the Cartesian coordinates) and $r$ is the aperture dependent scale factor.

For the elliptically polarized incident plane wave with the circular components

$$
E_{\nu}^{(\mathrm{inc})}=\exp \left(-i \nu \phi_{p}^{(\mathrm{inc})}\right)\left[1+\nu \epsilon_{\mathrm{ell}}^{\text {(inc) }}\right]\left|E_{\mathrm{inc}}\right|,
$$

expressed in terms of the polarization azimuth $\phi_{p}^{(\text {inc) }}$ and the ellipticity parameter $\epsilon_{\mathrm{ell}}^{(\mathrm{inc})}$, the reduced components of the transmitted wave are given by

$$
\begin{aligned}
E_{\nu}^{(\mathrm{tr})} /\left|E_{\mathrm{inc}}\right| \equiv & \Psi_{\nu}=\left|\Psi_{\nu}\right| e^{i \phi_{\nu}} \\
= & \left\{t_{\nu, \nu}\left[1+\nu \epsilon_{\mathrm{ell}}^{(\mathrm{inc})}\right]+t_{\nu,-\nu}\left[1-\nu \epsilon_{\mathrm{ell}}^{(\mathrm{inc})}\right]\right. \\
& \times \exp (-2 i \nu \psi)\} \exp \left(-i \nu \phi_{p}^{(\mathrm{inc})}\right),
\end{aligned}
$$

where $\psi=\phi-\phi_{p}^{(\text {inc })}$.

For the transmitted wave, the formula (58) gives the normalized Stokes parameters $s_{i}^{(\mathrm{tr})}=S_{i}^{(\mathrm{tr})} / S_{0}^{(\mathrm{tr})}$ as a function of the incidence angles $\theta_{\text {inc }}$ and $\phi_{\text {inc }}$. From Eqs. (51) and (52), these parameters are determined by the ellipticity $\epsilon_{\mathrm{ell}}=\left(\left|\Psi_{+}\right|\right.$ $\left.-\left|\Psi_{-}\right|\right) /\left(\left|\Psi_{+}\right|+\left|\Psi_{-}\right|\right)$, and the polarization azimuth $\phi_{p}=\left(\phi_{-}\right.$ $\left.-\phi_{+}\right) / 2$. Owing to the relation (56), the incidence angles and the points in the observation plane are in one-to-one correspondence. So, the distribution of the normalized Stokes parameters can be evaluated by computing the characteristics of the polarization ellipse $\epsilon_{\mathrm{ell}}$ and $\phi_{p}$ at each point of the projection plane. Geometrically, this procedure yields the two-dimensional field of polarization ellipses that might be called the polarization-resolved angular (conoscopic) pattern.

The point where $\left|\Psi_{\nu}\right|=0$ and thus the transmitted wave is circularly polarized with $\epsilon_{\mathrm{ell}}=-\nu$ will be referred to as the $C_{\nu}$ point. This is an example of the polarization singularity where the phases $\phi_{\nu}$ and $\phi_{p}$ become indeterminate.

Equivalently, $C$ points can be viewed as the phase singularities of the complex scalar field

$$
\tilde{S}=\Psi_{+}^{*} \Psi_{-}=\tilde{S}_{1}+i \tilde{S}_{2}
$$

proportional to the Stokes field defined in Eq. (51). Such singularities are characterized by the winding number which is the signed number of rotations of the two-component field $\left(\widetilde{S}_{1}, \widetilde{S}_{2}\right)$ around the circuit surrounding the singularity [2]. The winding number, also known as the signed strength of the dislocation, is generically \pm 1 .

Since the polarization azimuth (51) is defined modulo $\pi$ and $2 \phi_{p}=\arg \widetilde{S}$, the dislocation strength is twice the index of the corresponding $C_{\nu}$ point, $I_{C}$. For generic $C$ points, $I_{C}$ $= \pm 1 / 2$ and the index can be computed from the formula

$$
I_{C}=\frac{1}{2} \operatorname{sgn}\left[\operatorname{Im}\left(\partial_{x} \tilde{S}^{*} \partial_{y} \tilde{S}\right)\right]_{y=y_{\nu}}^{x=x_{\nu}}
$$

where $\partial_{x} f$ is the partial derivative of $f$ with respect to $x$.

The relation (60) gives the index of the $C_{\nu}$ point with the coordinates $\left(x_{\nu}, y_{\nu}\right)$ expressed in terms of the vorticity $[18,24]: \operatorname{Im}\left(\partial_{x} \tilde{S}^{*} \partial_{y} \widetilde{S}\right)=\partial_{x} \tilde{S}_{1} \partial_{y} \widetilde{S}_{2}-\partial_{y} \widetilde{S}_{1} \partial_{x} \tilde{S}_{2}$. The formula linking gradients of the complex field $(\mathbf{E}, \mathbf{E}) \propto \Psi_{+} \Psi_{-}$and the index for $C$ lines in the three-dimensional space was derived in Ref. [54].

In the case with $\Psi_{\nu}=0$ ( $C_{\nu}$ point $)$, only derivatives of $\Psi_{\nu}$ enter the expression (60), which can be suitably rearranged to yield the index of the $C_{\nu}$ point in the following form: 

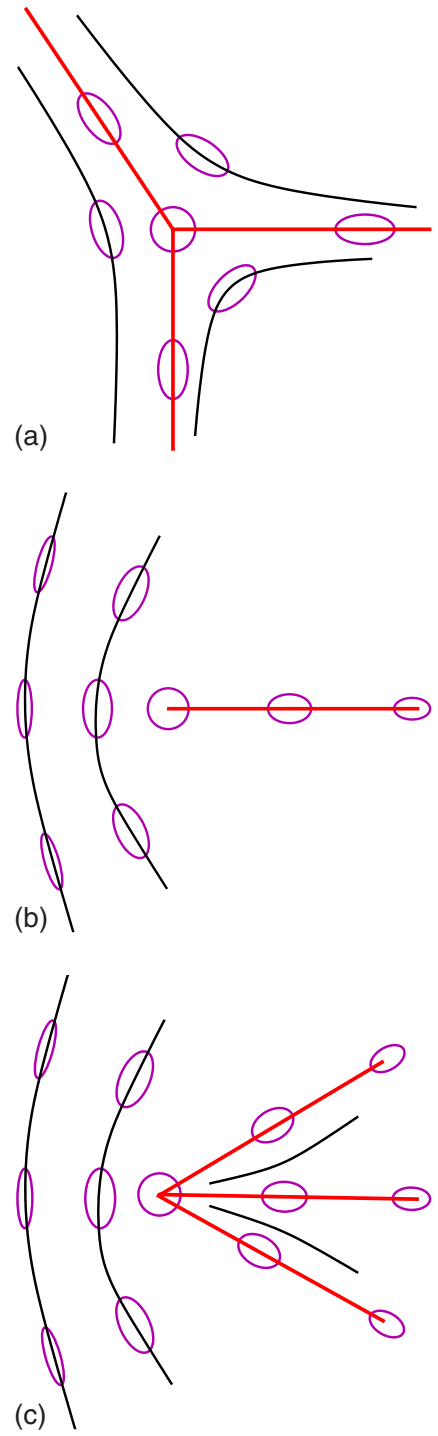

FIG. 3. (Color online) Arrangement of the polarization ellipses around the $C$ points of three different types. (a) Star: $I_{C}=-1 / 2$ and $N_{C}=3$. (b) Lemon: $I_{C}=+1 / 2$ and $N_{C}=1$. (c) Monstar: $I_{C}=+1 / 2$ and $N_{C}=3$.

$$
\begin{aligned}
I_{C} & =\frac{\nu}{2} \operatorname{sgn}\left[\operatorname{Im}\left(\partial_{x} \Psi_{\nu} \partial_{y} \Psi_{\nu}^{*}\right)\right]_{y=y_{\nu}}^{x=x_{\nu}} \\
& =\frac{\nu}{2} \operatorname{sgn}\left[\operatorname{Im}\left(\partial_{\rho} \Psi_{\nu} \partial_{\phi} \Psi_{\nu}^{*}\right)\right]_{\phi=\phi_{\nu}}^{\rho=\rho_{\nu}} .
\end{aligned}
$$

Subsequently, we shall apply the formula (61) expressing the index in terms of the derivatives with respect to polar coordinates to the case of a homeotropically aligned cell.

In addition to the handedness and the index, the $C$ points are classified according to the number of straight lines terminating on the singularity. This is the so-called line classification that was initially studied in the context of umbilic points [55].

For generic $C$ points, as is shown in Fig. 3, the number of the straight lines, $N_{C}$, may either be 1 or 3 . This number is 3 provided the index equals $-1 / 2, I_{C}=-1 / 2$, and such $C$ points are called stars. At $I_{C}=1 / 2$, there are two characteristic patterns of polarization ellipses around a $C$ point: (a) lemon with $N_{C}=1$ and (b) monstar with $N_{C}=3[10]$ (a monstar is a form on which three straight streamlines terminate). Different quantitative criteria to distinguish between the $C$ points of the lemon and the monstar types were deduced in Refs. $[24,30]$.

The location of the $C_{\nu}$ points on the projection plane is determined by the polar coordinates: $\rho_{k}^{(\nu)}$ and $\phi_{k}^{(\nu)}$, where $k$ is the numbering label. These can be found by solving the equation

$$
\left|\Psi_{\nu}(\rho, \phi)\right|=0
$$

that generally has multiple solutions.

The case of a linearly polarized wave with $\epsilon_{\mathrm{ell}}=0$ provides another example of the polarization singularity where the handedness is undefined. The curves along which the polarization is linear are called the L lines.

The transmitted wave is linearly polarized when the condition

$$
\left|\Psi_{+}(\rho, \phi)\right|=\left|\Psi_{-}(\rho, \phi)\right|
$$

is satisfied. So, Eq. (63) describes the loci of points forming the $L$ lines lying in the projection plane.

\section{Ellipticity induced effects}

\section{1. $C$ points: Creation and annihilation}

When the director is normal to the substrates, the NLC cell is homeotropically aligned and $d_{z}=1$. In this case the transmission matrix (43) is diagonal and its elements in the circular basis are given by

$$
t_{\nu, \nu}=t_{+}, \quad t_{\nu,-\nu}=t_{-}, \quad t_{ \pm}=\left(t_{e} \pm t_{o}\right) / 2,
$$

where the transmission coefficients

$$
t_{e, o}=t_{e, o}\left(q_{x}\right)=\left[\cos \left(\delta_{e, o}\right)-i \gamma_{e, o} \sin \left(\delta_{e, o}\right)\right]^{-1} \equiv t_{e, o}(\rho)
$$

are expressed in terms of the phases

$$
\delta_{e}=q_{z}^{(e)} h=n_{\perp} n_{\|}^{-1} \sqrt{n_{\|}^{2}-q_{x}^{2}} h, \quad \delta_{o}=q_{z}^{(o)} h=\sqrt{n_{\perp}^{2}-q_{x}^{2}} h,
$$

and the amplitudes $\gamma_{e, o}$ defined in Eq. (46).

Owing to the cylindrical symmetry of the homeotropic structure, the transmission coefficients (65) do not depend on the azimuthal angle of the incidence plane $\phi \equiv \phi_{\text {inc }}$. From Eq. (58) another consequence of this symmetry is that the azimuth $\phi_{p}-\phi_{p}^{\text {(inc) }}$, and the ellipticity of the transmitted wave depend only on the difference of the azimuthal angles: $\phi$ $-\phi_{p}^{(\text {inc) }}$. It follows that the sole effect of changing the polarization azimuth of the incident wave is the rotation of the polarization ellipse field by the angle $\phi_{p}^{\text {(inc) }}$. So, we focus our attention on the effects governed by the ellipticity of the incident wave, $\epsilon_{\mathrm{ell}}^{\text {(inc) }}$.

From Eqs. (58) and (64), the $C_{\nu}$ points may appear only if the condition 

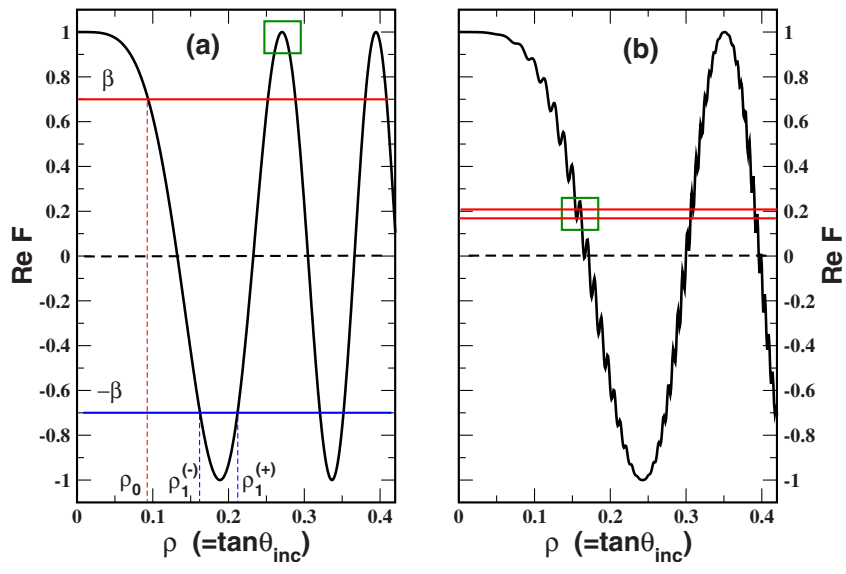

FIG. 4. (Color online) The real part of the function (69) that enters the left-hand side of Eq. (68) computed for (a) the cell filled with the NLC mixture E7 and (b) the KDP (potassium dihydrogen phosphate) birefringent crystal. The parameters are: (a) $n_{\perp}$ $=1.5246, n_{\|}=1.7608, n_{m}=1.5, d=110 \mu \mathrm{m}$, and (b) $n_{\perp}=1.5093, n_{\|}$ $=1.4683, n_{m}=1.0, d=300 \mu \mathrm{m}$.

$$
\frac{\left|t_{\nu,-\nu}\right|}{\left|t_{\nu, \nu}\right|} \equiv \frac{\left|t_{-}\right|}{\left|t_{+}\right|}=\frac{1+\nu \epsilon_{\mathrm{ell}}^{(\mathrm{inc})}}{1-\nu \epsilon_{\mathrm{ell}}^{\text {(inc) }}}
$$

is satisfied. Another form of this condition is

$$
\begin{gathered}
\operatorname{Re} F(\rho)=-\nu \frac{2 \epsilon_{\mathrm{ell}}^{(\mathrm{inc})}}{1+\left[\epsilon_{\mathrm{ell}}^{\text {(inc) }}\right]^{2}} \equiv \epsilon_{\mathrm{ell}} \mu_{\mathrm{inc}} \beta, \\
F(\rho)=\frac{2 t_{e}(\rho) t_{o}^{*}(\rho)}{\left|t_{e}(\rho)\right|^{2}+\left|t_{o}(\rho)\right|^{2}}=\frac{\exp (i \Delta)}{\cosh \left(\ln \left|t_{e}\right|-\ln \left|t_{o}\right|\right)},
\end{gathered}
$$

where $\beta=2\left|\epsilon_{\mathrm{ell}}^{(\mathrm{inc})}\right|\left(1+\left[\epsilon_{\mathrm{ell}}^{(\mathrm{inc})}\right]^{2}\right)^{-1}, \Delta=\arg \left(t_{e} t_{o}^{*}\right), \epsilon_{\mathrm{ell}}=-\nu$ is the ellipticity of the $C_{\nu}$ point, and $\mu_{\text {inc }}=\operatorname{sgn}\left(\epsilon_{\text {ell }}^{\text {(inc) }}\right)$ is the handedness (helicity) of the incident wave. Solutions of Eq. (68) define the radii of circles containing the $C$ points.

Plots depicted in Fig. 4 demonstrate nonmonotonic behavior of the function $\operatorname{Re} F$ that enter the left-hand side of Eq. (68).

The experimentally relevant case of the nematic cell filled with the LC mixture E7 is shown in Fig. 4(a). It is characterized by the weak anisotropy of the transmission amplitudes $\left|t_{e}\right|$ and $\left|t_{o}\right|$, so that $|1-| t_{e}|/| t_{o}||<10^{-4}$ for the incidence angles up to $60^{\circ}$. For such cells, the phase of the function (69), $\Delta$, can be approximated by the difference in optical path of the ordinary and extraordinary waves, $\delta=\delta_{e}-\delta_{o}$. So, the formula

$$
F(\rho) \approx F_{0}(\rho)=\exp (i \delta), \quad \delta=\delta_{e}-\delta_{o},
$$

provides a high accuracy approximation for the expression (69) and, for the LC mixture E7, the deviation $\max \left|F-F_{0}\right|$ can be estimated to be better than $5.0 \times 10^{-4}$.

The radii of the circles with the $C$ points can now be accurately located by solving the approximate equation $\cos \delta= \pm \beta$ obtained from Eq. (68) by using the expression (70). The result can be written as two sequences of radii,

$$
\begin{gathered}
\left|\delta\left(\rho_{0}\right)\right| \approx \arccos (\beta), \quad\left|\delta\left(\rho_{k}^{( \pm)}\right)\right| \approx \pm \arccos (\beta)+\pi k, \\
k=1, \ldots, N,
\end{gathered}
$$

where $N$ is determined by the number of solutions and the circles of each sequence, $\rho_{k}^{(+)}$and $\rho_{k}^{(-)}$, are numbered by the nonnegative integer $k$ in nondecreasing order of size, so that $\rho_{0} \equiv \rho_{0}^{( \pm)} \leqslant \rho_{1}^{(-)} \leqslant \rho_{1}^{(+)} \cdots \leqslant \rho_{k}^{(-)} \leqslant \rho_{k}^{(+)} \leqslant \cdots$.

At $\rho=\rho_{k}^{( \pm)}$, there is a pair of the $C$ points on each circle of the radius $\rho_{k}^{( \pm)}$with the ellipticity and the azimuthal angles given by

$$
\begin{gathered}
\left.\epsilon_{\mathrm{ell}}\right|_{\rho=\rho_{k}^{(\alpha)}}=(-1)^{k} \mu_{\mathrm{inc}} \equiv \mu_{k}, \quad k=0, \ldots, N, \\
\phi_{ \pm k}^{(\alpha)}=\phi_{p}^{\text {(inc) }} \pm \pi / 2-\frac{\mu_{k}}{2} \arg \left[t_{+}^{*} t_{-}\right]_{\rho=\rho_{k}^{(\alpha)}},
\end{gathered}
$$

where $\rho_{0}^{(\alpha)} \equiv \rho_{0}$ and $\alpha \in\{+,-\}$.

The symmetric arrangement of the $C$ points is a consequence of the symmetry relation

$$
\left|\Psi_{\nu}(\rho, \psi)\right|=\left|\Psi_{\nu}(\rho, \pi+\psi)\right|
$$

for the amplitudes (58).

We can now substitute Eq. (58) into the expression for the index (61) and use the relations (64) and (68) to recast the result into the form of the index formula

$$
I_{C}=-\frac{1}{2} \operatorname{sgn}\left[\partial_{\rho} \operatorname{Re} F(\rho)\right]_{\rho=\rho_{k}^{( \pm)}}=\frac{ \pm(-1)^{k}}{2}, \quad k=0, \ldots, N,
$$

where the second equality follows because $\operatorname{Re} F$ is an oscillating function of $\rho$ and $F(0)=1$.

Interestingly, Eq. (75) relates the index of $C$ points and derivatives of the transmission coefficients with respect to the incidence angle $\left(\partial_{\rho} f=\cos ^{2} \theta_{\text {inc }} \partial f / \partial \theta_{\text {inc }}\right)$. The index is determined by the circle number $k$ and alternates in sign starting from $I_{C}=+1 / 2$, which is the index of two $C$ points symmetrically arranged in the vicinity of the origin.

So, the pair of $C$ points lying on the smallest circle of the radius $\rho_{0}$ can either be of lemon or monstar types. Interestingly, when $\beta$ approaches unity and the incident light becomes circularly polarized, the radius $\rho_{0}$ vanishes and the $C$ points merge into one $C$ point of the index +1 .

This is the limiting case where the incident light is circular polarized with $\epsilon_{\mathrm{ell}}^{\text {inc) }}= \pm 1$ and a $C$ point may develop provided that the transmission coefficients meet the isotropy condition: $t_{e}= \pm t_{o}$. As can be seen from Eq. (65) we have $t_{e}=t_{o}$ for the case of normal incidence with $q_{x}=\sin \theta_{\text {inc }}=0$. The corresponding $C$ point is located at the origin and, as it was discussed earlier, its index $I_{C}$ equals +1 .

At this stage, however, it remains unclear if the abovementioned $C$ point is unique. In order to clarify this, we shall look more closely at the events that happen when the ellipticity parameter $\beta$ defined in Eq. (68) varies from zero to unity. At $\beta=0$, the incident light is in the state of linear polarization with $\epsilon_{\mathrm{ell}}^{(\mathrm{inc})}=0$, whereas the incident wave is circular polarized with $\left|\epsilon_{\text {ell }}^{(\text {inc) }}\right|=1$ at $\beta=1$.

The case of linearly polarized incident waves was previously studied in Refs. [29,30]. Figure 6(a) shows the 

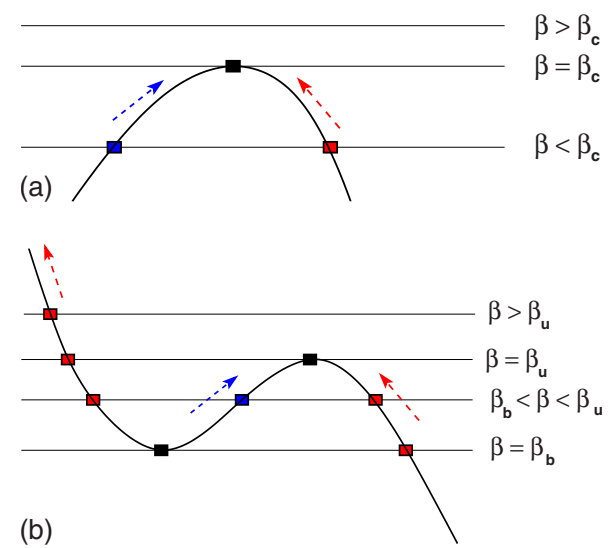

FIG. 5. (Color online) Schematic representation of creation and annihilation of $C$ points governed by saddle-node bifurcations of the solutions of Eq. (68) that take place within the areas marked by the boxes in Figs. 4(a) and 4(b). (a) $C$ points annihilate as the governing parameter $\beta$ passes through its critical value $\beta_{c}$ in the immediate vicinity of unity. (b) Two successive bifurcations result in creation and annihilation of $C$ points at $\beta=\beta_{b}$ and $\beta=\beta_{u}$, respectively.

polarization-resolved angular pattern as the polarization ellipse field computed at $\beta=0$. It can be seen that we have a sequence of $N+1$ concentric circles each containing two pairs of symmetrically arranged $C$ points. The index and the handedness of the $C$ points alternate in sign along the radial direction. The $C$ points on the circle of the smallest radius, $\rho=\rho_{0}$, corresponding to the direction close to the normal incidence are found to be of the lemon type with the index equal to $+1 / 2$.

Algebraically, from Eq. (71) it follows that the difference between the radii of the $C$ points $\rho_{k}^{(+)}$and $\rho_{k+1}^{(-)}$, where $k$ $=0, \ldots, N$, vanishes with the ellipticity $\epsilon_{\mathrm{ell}}^{\text {(inc) }}$ approaching zero. So, at $\epsilon_{\mathrm{ell}}^{(\mathrm{inc})}=0$, the angular polarization structure is characterized by the presence of degeneracy, which is a consequence of the additional symmetry relation

$$
\left|\Psi_{\nu}(\rho, \psi)\right|=\left|\Psi_{-\nu}(\rho, \pi-\psi)\right| .
$$

The symmetry-breaking effects due to nonvanishing ellipticity of the incident wave are illustrated in Figs. 6(b) and 6(c) for small values of $\epsilon_{\mathrm{ell}}^{\text {(inc) }}$. This is the case when the relation (76) breaks down removing the above degeneracy, so that $\rho_{k}^{(+)}<\rho_{k+1}^{(-)}$at $\epsilon_{\mathrm{ell}}^{(\mathrm{inc})} \neq 0$.

As is schematically illustrated by Fig. 5(a), there is another type of degeneracy which takes place when the ellipticity parameter $\beta$ reaches a local maximum of the function $\operatorname{Re} F$ such as the one marked by a box in Fig. 4(a). In contrast to the above-discussed case of linear polarization, the number of $C$ points changes as the parameter $\beta$ and the ellipticity $\epsilon_{\mathrm{ell}}^{\text {(inc) }}$ pass through their critical values $\beta_{c}$ and $\epsilon^{(c)}$.

The picture shown in Fig. 5(a) is typical of a saddle-node (tangent) bifurcation [56,57]. Such bifurcations occur at the incidence angles represented by the values of the radii [see Eq. (56)] at local extrema of the real part of the function (69). By applying the approximation (70), these radii can be estimated from the solutions of the equation

$$
\left|\delta\left(\rho_{k}^{(c)}\right)\right| \approx \pi k, \quad k=0, \ldots, N,
$$

obtained from Eq. (71) by setting $\arccos (\beta)=0$.

Given the critical value of the radius, $\rho=\rho_{k}^{(c)}$, we can use Eq. (68) to deduce the relations

$$
\beta_{k}^{(c)}=\left|\operatorname{Re} F\left(\rho_{k}^{(c)}\right)\right|, \quad \beta_{k}^{(c)} \epsilon_{k}^{(c)}=-1+\sqrt{1-\left[\beta_{k}^{(c)}\right]^{2}},
$$

and to compute the corresponding values of the parameter $\beta$ and the ellipticity: $\beta=\beta_{k}^{(c)}$ and $\left|\epsilon_{\mathrm{ell}}^{(\mathrm{inc})}\right|=\epsilon_{k}^{(c)}$. It is also not difficult to show that $\rho_{0}^{(c)}=0$ and $\rho_{k}^{(-)} \leqslant \rho_{k}^{(c)} \leqslant \rho_{k}^{(+)}$

Our analysis suggests that the ellipse field undergoes a sequence of tangent bifurcations as the magnitude of the ellipticity $\left|\epsilon_{\mathrm{ell}}^{(\mathrm{inc})}\right|$ increases from zero to unity. There are two pairs of the $C$ points with $\rho=\rho_{k}^{(-)}$and $\rho=\rho_{k}^{(+)}$that merge and, subsequently, annihilate as the governing parameter passes through the bifurcation point with $\left|\epsilon_{\mathrm{ell}}^{(\mathrm{inc})}\right|=\epsilon_{k}^{(c)}$ and $\rho_{k}^{(+)}=\rho_{k}^{(-)}$.

In addition, an important consequence of the index formula (75) is that the indices of the $C$ points involved in a tangent bifurcation differ only in sign, so that the total sum of indices remains intact. Obviously, this result agrees with the conservation law of the total topological index.

Note, however, that, within the approximation (70), the $C$ points cannot merge and annihilate provided the incident wave is not circular polarized. Mathematically, the difficulty is that, for the approximate function $\cos \delta$, the critical values of the ellipticity are all equal to unity, $\beta_{k}^{(c)}=\epsilon_{k}^{(c)}=1$. So, we arrive at the conclusion that the difference $1-\epsilon_{k}^{(c)}$ is determined by the accuracy of the approximation (70).

For the cell filled with the LC mixture E7, the first three critical values of the ellipticity $\epsilon_{3}^{(c)}<\epsilon_{2}^{(c)}<\epsilon_{1}^{(c)}$ turned out to be very close to unity: $1-\epsilon_{1}^{(c)} \approx 1.22 \times 10^{-6}, 1-\epsilon_{2}^{(c)} \approx 1.17$ $\times 10^{-5}$, and $1-\epsilon_{3}^{(c)} \approx 4.16 \times 10^{-5}$. The polarization-resolved angular patterns presented in Fig. 7 are computed at the three different values of $\epsilon_{\mathrm{ell}}^{\text {(inc) }}$ : (a) $\epsilon_{3}^{(c)}<\epsilon_{\mathrm{ell}}^{\text {(inc) }}=\epsilon_{2}^{(c)}-10^{-7}<\epsilon_{2}^{(c)}$; (b) $\epsilon_{2}^{(c)}<\epsilon_{\mathrm{ell}}^{(\mathrm{inc})}=\epsilon_{1}^{(c)}-10^{-7}<\epsilon_{1}^{(c)} ; \quad(\mathrm{c}) \quad \epsilon_{1}^{(c)}<\epsilon_{\mathrm{ell}}^{(\mathrm{inc})}=\epsilon_{1}^{(c)}+10^{-6}<1$. The pairs of the $C$ points with $k=3, k=2$, and $k=1$ are shown to coalesce and disappear in succession as the ellipticity passes sequentially through $\epsilon_{3}^{(c)}, \epsilon_{2}^{(c)}$, and $\epsilon_{1}^{(c)}$.

So, in the limit of circular polarization with $\epsilon_{\mathrm{ell}}^{(\mathrm{inc})}=1$, we have the pattern where the only $C$ point is located at the origin. The index of this $C$ point is $I_{C}=+1$. This result is in agreement with the Poincaré-Hopf theorem linking the Euler characteristics $\chi$ of a two-dimensional manifold and the total topological charge (index) of a smooth vector field on the manifold $[8,58]$. In our case, it can be concluded that $\chi$ $=2 I_{C}$ is the total index, where $\chi=+2$ is the Euler characteristics of a sphere.

So far, we considered bifurcations at the local extrema that, according to Eq. (77), are in one-to-one correspondence with the extrema of the function $\operatorname{Re} F_{0}=\cos \delta$. Thus, such bifurcations are governed by the phase difference between ordinary and extraordinary waves, $\delta=\delta_{e}-\delta_{o}$. As is shown in Fig. 7 , they are typically characterized by extremely small differences between unity and the critical values of the ellipticity. For this reason they cannot be detected experimentally.

From the above discussion it can be inferred that the critical values might be lowered only if the approximation (70) does not work well. In particular, this is the case for the 
graph of Re $F$ plotted in Fig. 4(b) where the anisotropic material is represented by the KDP (potassium dihydrogen phosphate) birefringent crystal. The deviation $\max \left|F-F_{0}\right|$ can be estimated at about $7.0 \times 10^{-2}$, so that $\max \left[1-\epsilon_{k}^{(c)}\right]$ $\approx 0.02$.

The curve shown in Fig. 4(b) also demonstrates that the breakdown of the approximation (70) may manifest itself in additional extrema formed away from the neighborhood of unity. As is illustrated in Fig. 5(b) for the region marked by the box in Fig. 4(b), these local extrema result in two successive tangent bifurcations leading to creation and annihilation of two different pairs of the $C$ points.

In contrast to the extrema described by the phase difference $\delta$, this is the multireflection which is primarily responsible for additional small-scale oscillations. Typically, it is a challenging task to resolve accurately these ripplelike and noisy oscillations in polarimetry measurements.

\section{Avoided L-line crossings}

Now we pass on to discussing the $L$ lines which are the curves of linear polarization in the observation plane. Analytically, substituting Eq. (58) into the relation (63) and using the matrix elements (64) give the equation

$$
2 \epsilon_{\mathrm{ell}}^{(\mathrm{inc})} \operatorname{Re} F(\rho)+\left(1-\left[\epsilon_{\mathrm{ell}}^{(\mathrm{inc})}\right]^{2}\right) \sin (2 \psi) \operatorname{Im} F(\rho)=0,
$$

describing $L$ lines for the case of the homeotropically aligned NLC cell.

We begin with the results for the case of linearly polarized incident waves studied in Refs. $[29,30]$. At $\epsilon_{\mathrm{ell}}^{(\mathrm{inc})}=0$, from Eq. (79) there are two straight lines of linear polarization: $\phi$ $=\phi_{p}^{\text {(inc) }}$ and $\phi=\phi_{p}^{\text {(inc) }}+\pi / 2$, where the polarization vectors of incident and transmitted waves are parallel, $\phi_{p}^{(\mathrm{tr})} \equiv \phi_{p}$ $=\phi_{p}^{\text {(inc) }}$. Other $L$ lines are circles separating the circles of $C$ points. Interestingly, the radii of the circles, $\rho_{k}^{(L)}$, can be found as the solutions of Eq. (77) with $k=1, \ldots, N$.

When $k$ is even, it can be concluded that, similar to the straight $L$ lines, $\phi_{p} \approx \phi_{p}^{(\text {inc) }}$. If $k$ is odd, the polarization vector of the transmitted wave rotates with the azimuthal angle of the incidence plane and $\phi_{p} \approx \phi_{p}^{(\text {inc) }}+2 \phi$.

The structure of the $L$ lines at $\beta=0$ is shown in Fig. 6(a), where the $L$ lines are represented by the solid lines bounding the regions of left- and right-handed polarization. As it was discussed in the preceding section, the case of linear polarization possesses additional symmetry described by the relation (76). This symmetry is broken at nonzero values of the ellipticity and the case of linear polarization is structurally unstable.

In Figs. 6(b) and 6(c), we show that even small values of the ellipticity, $\epsilon_{\mathrm{ell}}^{\text {(inc) }}$, change the structure of the intersecting $L$ lines depicted in Fig. 6(a) into a family of nonintersecting closed $L$ lines. The figures clearly demonstrate what might be called, by analogy with the well-known avoided level crossings in the quantum mechanics, the effect of avoided L-line crossings.

The closed $L$ lines gradually evolve into the circles as the ellipticity approaches the limit of circular polarization with $\left|\epsilon_{\text {ell }}^{(\text {inc })}\right|=1$. The radii of the $C$ circles $\rho_{k}^{(L)}$ can be found by solving the equation $\operatorname{Re} F(\rho)=0$. It follows that $\rho_{k}^{(-)}$at $\epsilon_{\mathrm{ell}}^{\text {(inc) }}$

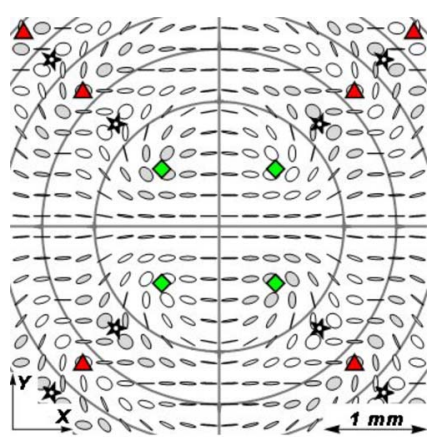

(a)

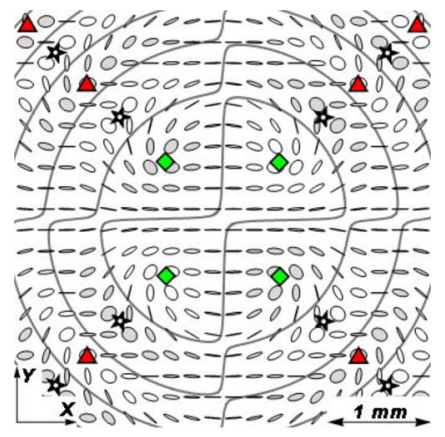

(b)

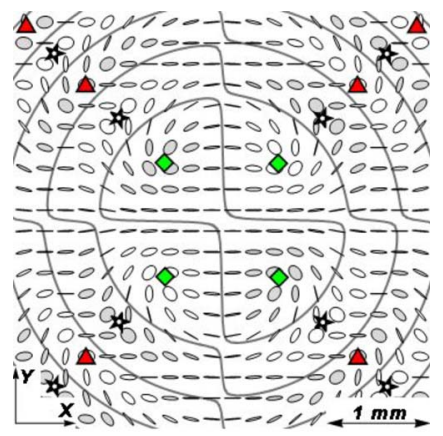

(c)

FIG. 6. (Color online) Polarization-resolved conoscopic patterns computed as polarization ellipse fields in the observation plane for the homeotropically oriented cell filled with the NLC mixture E7 at small values of the ellipticity: (a) $\epsilon_{\mathrm{ell}}^{\text {(inc) }}=0.0$, (b) $\epsilon_{\mathrm{ell}}^{\text {(inc) }}=0.01$, and (c) $\epsilon_{\mathrm{ell}}^{(\mathrm{inc})}=-0.01$. The parameters used in calculations are as follows: the wavelength is $\lambda_{\mathrm{inc}}=632.8 \mathrm{~nm}$; the polarization azimuth is $\phi_{p}^{(\mathrm{inc})}=0$; the cell thickness is $d=110 \mu \mathrm{m}$; the scale factor is $r=5 \mathrm{~mm} ; n_{m}$ $=1.5, n_{\perp}=1.5246$, and $n_{\|}=1.7608$. The $C$ points of the star, the lemon, and the monstar types are marked by stars, diamonds, and triangles, respectively. $L$ lines are represented by solid lines. Lefthanded and right-handed polarization is, respectively, indicated by solid and open ellipses.

$=0$ is equal to $\rho_{k}^{(L)}$ at $\left|\epsilon_{\mathrm{ell}}^{(\mathrm{inc})}\right|=1$. So, we have a family of the concentric circles describing both the $C$ points for linearly polarized incident waves [see Fig. 6(a)] and the $L$ lines for circular polarized incident waves (see Fig. 7).

\section{RESULTS AND DISCUSSION}

The analytical results presented in the previous section completely characterize the polarization-resolved angular 


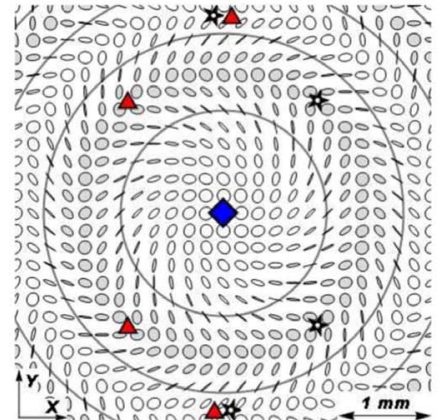

(a)

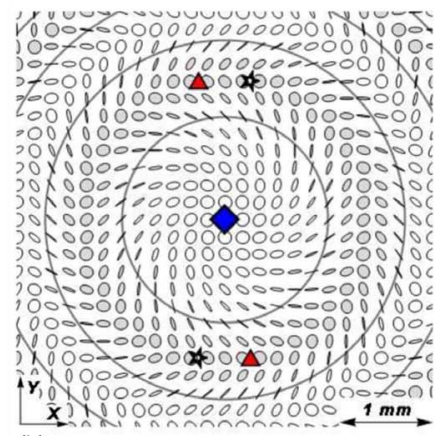

(b)

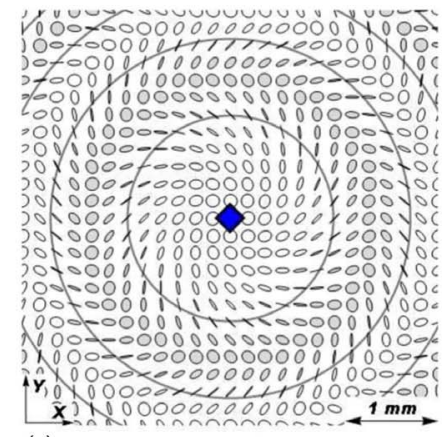

(c)

FIG. 7. (Color online) Polarization-resolved conoscopic patterns of the homeotropically oriented NLC cell computed at different values of the ellipticity. Three cases are shown: (a) $\epsilon_{3}^{(c)}<\epsilon_{\text {ell }}^{\text {(inc) }}$ $<\epsilon_{2}^{(c)}$; (b) $\epsilon_{2}^{(c)}<\epsilon_{\mathrm{ell}}^{(\mathrm{inc})}<\epsilon_{1}^{(c)}$; (c) $\epsilon_{1}^{(c)}<\epsilon_{\mathrm{ell}}^{(\mathrm{inc})}<1$. Other parameters are listed in the caption of Fig. 6.

patterns of light transmitted through the NLC cell for the homeotropic orientational structure in terms of the polarization singularities. These patterns can be conveniently represented as the fields of polarization ellipses and characterize the polarization structure behind the conoscopic images measured in experiments with two crossed polarizers.

After computing the loci of the $C$-points and the $L$ lines, we have deduced a simple formula for the index of the $C$ points (75) and studied what happens to the polarization singularities if the ellipticity and the polarization azimuth of the incident wave vary. It turned out that the $C$ points may appear and disappear when the pattern undergoes bifurcations at some critical values of the ellipticity. In addition, the patterns formed in the neighborhood of zero ellipticity (linearly polarized incident light) are characterized by the appearance of avoided $L$-line crossings.

Now we compare the experimentally measured and the theoretically calculated polarization-resolved angular pat-

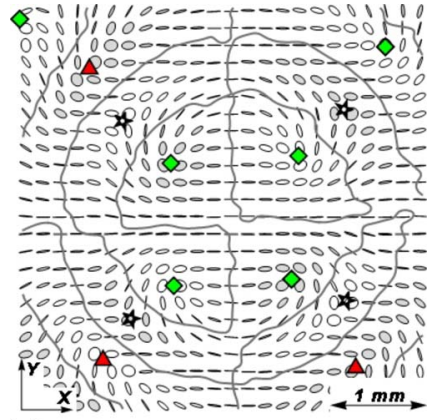

(a)

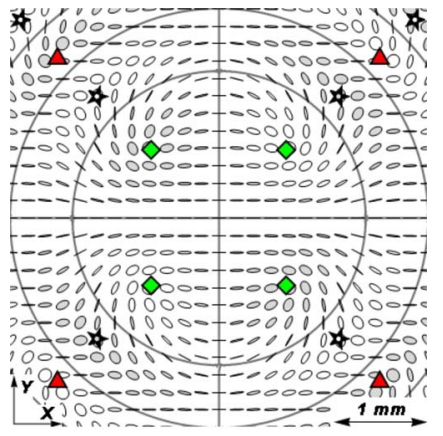

(b)

FIG. 8. (Color online) (a) Experimentally measured and (b) theoretically computed fields of polarization ellipses in the observation plane for the homeotropically aligned cell of the thickness $d=110 \mu \mathrm{m}$ filled with the NLC mixture E7. The incident wave is linearly polarized with the polarization azimuth $\phi_{p}^{\text {(inc) }}=0$. The $C$ points of the star, the lemon, and the monstar types are marked by stars, diamonds, and triangles, respectively. $L$ lines are represented by solid lines. Left-handed and right-handed polarization is, respectively, indicated by solid and open ellipses.

terns for the homeotropically oriented NLC cell. These patterns are shown in Figs. 8-10 as the fields of polarization ellipses in the observation plane where the polar coordinates are defined in Eq. (56) and the scale factor $r$ is taken to be $5 \mathrm{~mm}$. The parameters used in our calculations are described in Sec. II. They are also listed in the caption of Fig. 6.

In Fig. 8, we present the results obtained for the linearly polarized wave with $\epsilon_{\mathrm{ell}}^{(\mathrm{inc})}=0.0$, whereas the cases of elliptically polarized incident light with $\epsilon_{\mathrm{ell}}^{(\mathrm{inc})}=0.26$ and $\epsilon_{\mathrm{ell}}^{\text {(inc) }}$ $=0.63$ are shown in Figs. 9 and 10, respectively. As is evident from the figures, the $C$ points are arranged in chains formed by four rays along which they alternate in sign of the handedness and of the index. The $L$ lines are typically represented by the closed curves separating the regions of different polarization handedness. It is also clear that the predictions of the theory discussed in Sec. III C are in good agreement with the experimental data.

In addition, by making a comparison between the patterns with different values of ellipticity we arrive at the conclusion that, when the ellipticity changes, the $C$ points mainly move along the radial direction and their azimuthal angles remain approximately constant. Theoretically, it can be shown that, at $\phi_{p}^{(\text {inc) }}=0$, the azimuthal angle $\left|\phi_{ \pm k}^{(\alpha)}\right|$ given by Eq. (73) does not depart appreciably from $\pi / 4$ provided the magnitude of the ellipticity $\left|\epsilon_{\mathrm{ell}}^{\text {(inc) }}\right|$ is not in the immediate vicinity of unity. 

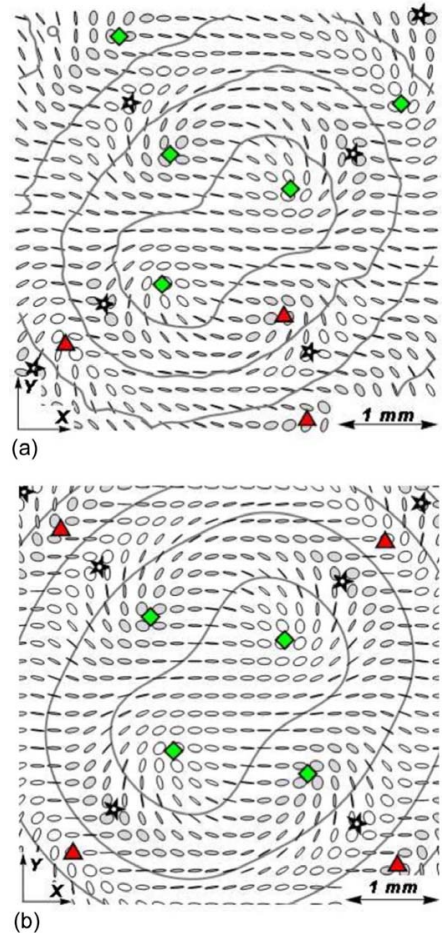

FIG. 9. (Color online) (a) Experimentally measured and (b) theoretically computed fields of polarization ellipses for the homeotropically aligned cell filled with the NLC mixture E7. The ellipticity of the incident light is $\epsilon_{\mathrm{ell}}^{(\mathrm{inc})}=0.26$.

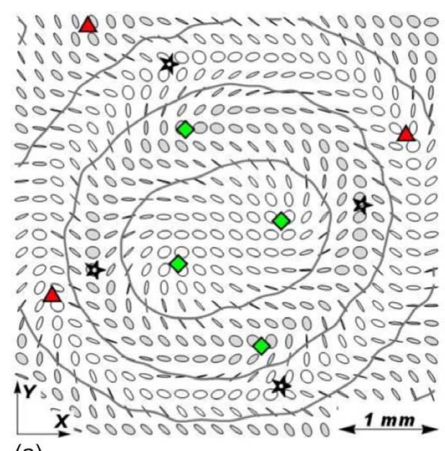

(a)

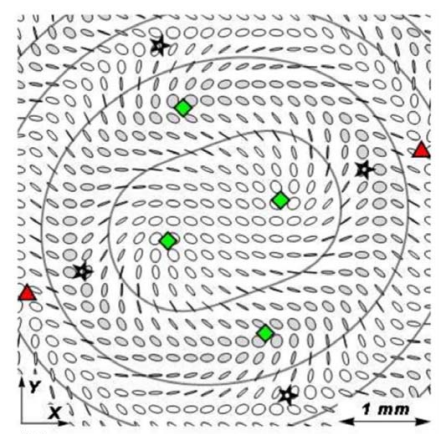

FIG. 10. (Color online) (a) Experimentally measured and (b) theoretically computed fields of polarization ellipses for the homeotropically aligned cell filled with the NLC mixture E7. The ellipticity of the incident wave is $\epsilon_{\mathrm{ell}}^{(\mathrm{inc})}=0.63$ and the polarization azimuth is $\phi_{p}^{\text {(inc) }} \approx 20^{\circ}$.

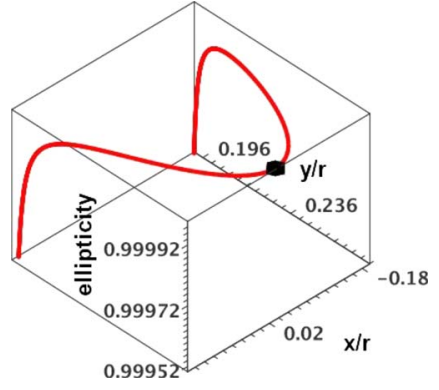

FIG. 11. (Color online) Right-handed $C$ lines near the bifurcation point with $\epsilon_{\mathrm{ell}}^{(\mathrm{inc})}=\epsilon_{2}^{(c)}$ in $\left(x, y, \epsilon_{\mathrm{ell}}^{(\mathrm{inc})}\right)$ space. $C$ points annihilate at the bifurcation point $\left(0, \rho_{2}^{(c)}, \epsilon_{2}^{(c)}\right)$, marked by a black solid diamond [see also Fig. 7(a)]. Curves are computed for the homeotropically aligned cell filled with the NLC mixture E7.

By contrast, as is shown in Fig. 7, annihilation of the $C$ points is accompanied by drastic changes in the azimuth of the $C$ points, whereas the radius is very close to its critical value defined in Eq. (77).

In the pseudo-three-dimensional $\left(x, y, \epsilon_{\mathrm{ell}}^{\text {(inc) }}\right)$ space, where the coordinates in the plane of observation, $x$ and $y$, are augmented by the incident wave ellipticity giving the third dimension, this effect manifests itself in the specific type of local structure of $C$ lines close to the bifurcation point depicted in Fig. 11. In particular, the $C$ curve is shown to sharply bend toward the bifurcation point. Such bends can be seen as cusps in the global structure of entwining $C$ lines presented in Fig. 12.

Figures 11 and 12 suggest that the geometric rearrangements and transformations studied in this paper might be regarded as pseudodynamics of the polarization ellipse fields with the ellipticity served as a "time" parameter. In threedimensional space, phase singularity lines, whose geometry depends on a time parameter, were recently investigated in Ref. [59].

In conclusion, we note that the homeotropic alignment presents the simplest case of anisotropic orientational struc-

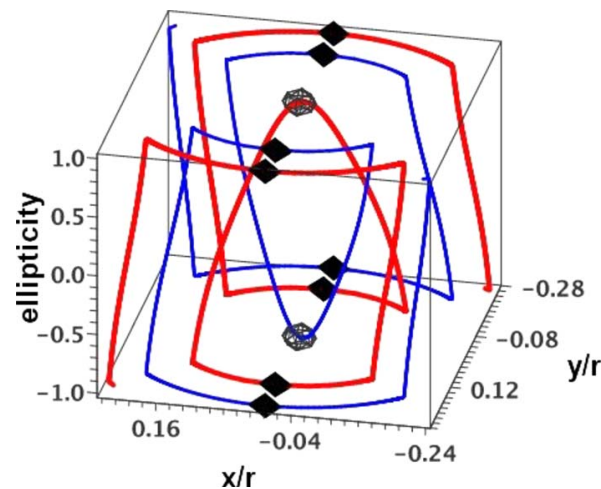

FIG. 12. (Color online) $C$ lines in the three-dimensional $\left(x, y, \epsilon_{\mathrm{ell}}^{\text {(inc) }}\right)$ space computed for the homeotropically aligned cell filled with the NLC mixture E7. Bifurcation points where $C$ points annihilate are shown as black solid diamonds. Two circular points, $(0,0,1)$ and $(0,0,-1)$, indicate the $C$ points with the index $I_{C}$ $=+1$ formed at circularly polarized incident wave. Right- and lefthanded $C$ points are represented by thick and thin lines, respectively. 
ture. Our theoretical analysis can be extended to more complicated cases involving both uniaxial and biaxial anisotropies by using the analytical results presented in the Appendix. These results will be published elsewhere.

\section{ACKNOWLEDGMENTS}

This work was partially supported by HKUST CERG Grant No. 612406 and by STCU Grant No. 4687.

\section{APPENDIX: OPERATOR OF EVOLUTION}

Equations (36) and (38) express the transmission and reflection matrices in terms of the linking matrix (34), which is determined by the evolution operator (26). In the case of uniform anisotropy, the operator can be computed as the evolution matrix (39) expressed in terms of the eigenvalues and the eigenvectors (40) of the matrix (25).

In this section we consider how the eigenvalue problem can be solved using the basis of eigenmodes (normal modes) in a uniformly anisotropic medium. These eigenmodes are known to be linearly polarized plane waves characterized by the $\hat{\mathbf{k}}$ dependent refractive indices $[44,52,60]$.

\section{Orthogonality relations}

From Eqs. (25a)-(25d), if the dielectric tensor $\boldsymbol{\varepsilon}$ is symmetric, $\epsilon_{i j}=\epsilon_{j i}$, we deduce the relations for the block matrices

$$
\mathbf{M}_{12}^{T}=\mathbf{M}_{12}, \quad \mathbf{M}_{21}^{T}=\mathbf{M}_{21}, \quad \mathbf{M}_{11}^{T}=\mathbf{M}_{22},
$$

giving the symmetry identity

$$
\mathbf{M}^{T}=\mathbf{G} \cdot \mathbf{M} \cdot \mathbf{G}, \quad \mathbf{G}=\left(\begin{array}{cc}
\mathbf{0} & \mathbf{I}_{2} \\
\mathbf{I}_{2} & \mathbf{0}
\end{array}\right)
$$

for the matrix $\mathbf{M}$.

The left eigenvectors $\left\{\mathbf{V}_{1}^{\prime}, \mathbf{V}_{2}^{\prime}, \mathbf{V}_{3}^{\prime}, \mathbf{V}_{4}^{\prime}\right\}$ of the matrix $\mathbf{M}$ can be defined as the right eigenvectors of its transpose $\mathbf{M}^{T}$,

$$
\mathbf{M}^{T} \cdot \mathbf{V}_{i}^{\prime}=\lambda_{i} \mathbf{V}_{i}^{\prime} \Leftrightarrow \mathbf{M}^{T} \cdot \mathbf{V}^{\prime}=\mathbf{V}^{\prime} \cdot \Lambda
$$

where $\mathbf{V}^{\prime}=\left(\mathbf{V}_{1}^{\prime} \mathbf{V}_{2}^{\prime} \mathbf{V}_{3}^{\prime} \mathbf{V}_{4}^{\prime}\right)$ is the matrix of the left eigenvectors. It is not difficult to prove that the left and right eigenvector form a biorthogonal set

$$
\left(\mathbf{V}_{i}^{\prime}, \mathbf{V}_{j}\right)=\left[\mathbf{V}_{i}^{\prime}\right]^{T} \cdot \mathbf{V}_{j}=\delta_{i j} N_{i} \Leftrightarrow \mathbf{V}^{\prime T} \cdot \mathbf{V}=\mathbf{N}
$$

where $\mathbf{N}=\operatorname{diag}\left(N_{1}, N_{2}, N_{3}, N_{4}\right)$.

Equation (A2) and the definition (A3) can now be combined to yield the relation

$$
\mathbf{V}^{\prime}=\mathbf{G} \cdot \mathbf{V},
$$

linking the matrices of the left and right eigenvectors, $\mathbf{V}^{\prime}$ and V. It remains to notice that the identities (A4) and (A5) give both the orthogonality relation

$$
\mathbf{V}^{T} \cdot \mathbf{G} \cdot \mathbf{V}=\mathbf{N} \equiv \operatorname{diag}\left(N_{1}, N_{2}, N_{3}, N_{4}\right)
$$

and the formula for inverse of the eigenvector matrix

$$
\mathbf{V}^{-1}=\mathbf{N}^{-1} \cdot \mathbf{V}^{T} \cdot \mathbf{G}
$$

The relations given in Eqs. (31) and (32) represent the special case of isotropic media and, thus, immediately follow from Eqs. (A6) and (A7).

By combining the identities (32) and (A7) with the expression for the evolution operator (39), we derive the linking matrix (34) for the case of uniform anisotropy in the following form:

$$
\begin{gathered}
\mathbf{W}=\mathbf{V}_{m}^{-1} \cdot \exp \{-i \mathbf{M} h\} \cdot \mathbf{V}_{m}=\mathbf{N}_{m}^{-1} \cdot \tilde{\mathbf{W}} \\
=N_{m}^{-1}\left(\begin{array}{cc}
\tilde{\mathbf{W}}_{11} & \tilde{\mathbf{W}}_{12} \\
-\tilde{\mathbf{W}}_{21} & -\tilde{\mathbf{W}}_{22}
\end{array}\right), \\
\tilde{\mathbf{W}}=\tilde{\mathbf{V}}^{T} \cdot \mathbf{W}_{d} \cdot \tilde{\mathbf{V}}, \\
\tilde{\mathbf{V}}=\mathbf{V}^{T} \cdot \mathbf{G} \cdot \mathbf{V}_{m}, \quad \mathbf{W}_{d}=\mathbf{N}^{-1} \cdot \exp \{-i \boldsymbol{\Lambda} h\} .
\end{gathered}
$$

From Eqs. (A8) and (A9) the matrices $\widetilde{\mathbf{W}}$ and $\mathbf{W}_{i i}$ are symmetric, whereas $\mathbf{W}_{21}^{T}=-\mathbf{W}_{12}$. So, we conclude that the transmission matrix $\mathbf{T}=\mathbf{W}_{11}^{-1}$ is symmetric too.

Note that, for a nonabsorbing medium characterized by a real matrix $\mathbf{M}, \operatorname{Im} \mathbf{M}=\mathbf{0}$, inverse of the matrix (A8) can be derived by complex conjugation: $\mathbf{W}^{-1}=\mathbf{W}^{*}$, where an asterisk indicates complex conjugation. In particular, the conservation law

$$
\mathbf{T}^{\dagger} \cdot \mathbf{T}+\mathbf{R}^{\dagger} \cdot \mathbf{R}=\mathbf{I}_{2},
$$

where the superscript $\dagger$ stands for Hermitian conjugation, can be immediately deduced from the relation

$$
\mathbf{W}_{11}^{*} \cdot \mathbf{W}_{11}+\mathbf{W}_{12}^{*} \cdot \mathbf{W}_{21}=\mathbf{W}_{11}^{\dagger} \cdot \mathbf{W}_{11}-\mathbf{W}_{21}^{\dagger} \cdot \mathbf{W}_{21}=\mathbf{I}_{2} .
$$

\section{Uniformly biaxial media}

For plane waves, owing to the Maxwell equation (8b), the electric displacement field is transverse, $(\mathbf{D}, \mathbf{k})=0$. So, assuming that the wave vector $\mathbf{k}$ lies in the plane of incidence (the $x-z$ plane), the vector $\mathbf{D}$ can be conveniently defined by its components in the basis $\left\{e_{1}(\hat{\mathbf{k}}), e_{2}(\hat{\mathbf{k}}), e_{0}(\hat{\mathbf{k}}) \equiv \hat{\mathbf{k}}\right\}$,

$$
\begin{gathered}
\mathbf{D}=D_{1} e_{1}(\hat{\mathbf{k}})+D_{2} e_{2}(\hat{\mathbf{k}}), \\
\hat{\mathbf{k}} \equiv e_{0}(\hat{\mathbf{k}})=k^{-1} \mathbf{k}=q^{-1}\left(q_{x} \hat{\mathbf{x}}+q_{z} \hat{\mathbf{z}}\right),
\end{gathered}
$$

where $e_{1}(\hat{\mathbf{k}})=q^{-1}\left(q_{z} \hat{\mathbf{x}}-q_{x} \hat{\mathbf{z}}\right), e_{2}(\hat{\mathbf{k}})=\hat{\mathbf{y}}$, and $q=k / k_{\mathrm{vac}}=n$ is the refractive index.

Given the electric displacement field (A13), the electric field can be found from the constitutive relation

$$
\begin{gathered}
\mathbf{E}=\mu \boldsymbol{\eta} \cdot \mathbf{D}, \\
\boldsymbol{\eta}=\eta_{3} \mathbf{I}_{3}+\Delta \eta_{1} \hat{\mathbf{d}} \otimes \hat{\mathbf{d}}+\Delta \eta_{2} \hat{\mathbf{m}} \otimes \hat{\mathbf{m}},
\end{gathered}
$$

where $\eta_{i}=\left(\mu \epsilon_{i}\right)^{-1}, \Delta \eta_{i}=\eta_{i}-\eta_{3}$, and $\mu \boldsymbol{\eta}$ is the inverse dielectric tensor $\left(\mu \boldsymbol{\eta} \cdot \boldsymbol{\varepsilon}=\mathbf{I}_{3}\right)$. 
The Maxwell equations can now be combined with the relation (A15) to yield the equation for the displacement vector $\mathbf{D}$ in the form of an eigenvalue problem

$$
\boldsymbol{\eta}_{t} \cdot \mathbf{D}=q^{-2} \mathbf{D}, \quad \boldsymbol{\eta}_{t}=\mathbf{P}(\hat{\mathbf{k}}) \cdot \boldsymbol{\eta} \cdot \mathbf{P}(\hat{\mathbf{k}}),
$$

where $\mathbf{P}(\hat{\mathbf{k}})=\mathbf{I}_{3}-\hat{\mathbf{k}} \otimes \hat{\mathbf{k}}$ is the projector onto the plane normal to the wave vector $\mathbf{k}$. By using the expression for the inverse dielectric tensor (A16) we can rewrite Eq. (A17) in the explicit matrix form as follows:

$$
\begin{gathered}
{\left[a_{-} \boldsymbol{\sigma}_{3}+b \boldsymbol{\sigma}_{1}-c \mathbf{I}_{2}\right]\left(\begin{array}{l}
D_{1} \\
D_{2}
\end{array}\right)=\mathbf{0}, \quad \boldsymbol{\sigma}_{1}=\left(\begin{array}{ll}
0 & 1 \\
1 & 0
\end{array}\right),} \\
2 a_{\mp}=\Delta \eta_{1}\left(\tilde{d}_{1}^{2} \mp \widetilde{d}_{2}^{2}\right)+\Delta \eta_{2}\left(\tilde{m}_{1}^{2} \mp \tilde{m}_{2}^{2}\right) \\
b=\Delta \eta_{1} \tilde{d}_{1} \tilde{d}_{2}+\Delta \eta_{2} \tilde{m}_{1} \tilde{m}_{2} \\
c=1-\eta_{3} q^{2}-a_{+},
\end{gathered}
$$

where $\tilde{d}_{i}=q\left(\hat{\mathbf{d}}, e_{i}(\hat{\mathbf{k}})\right)$ and $\tilde{m}_{i}=q\left(\hat{\mathbf{m}}, e_{i}(\hat{\mathbf{k}})\right)$. Then the dispersion relation (Fresnel's equation)

$$
\begin{gathered}
{\left[1-\eta_{3} q^{2}\right]\left\{1-\eta_{3} q^{2}-\Delta \eta_{1}\left[q^{2}-(\hat{\mathbf{d}}, \mathbf{q})^{2}\right]-\Delta \eta_{2}\left[q^{2}-(\hat{\mathbf{m}}, \mathbf{q})^{2}\right]\right\}} \\
-\Delta \eta_{1} \Delta \eta_{2} q^{2}(\hat{\mathbf{d}} \times \hat{\mathbf{m}}, \mathbf{q})^{2}=0
\end{gathered}
$$

can be derived as the condition for the system of linear equations (A18) to have a nonvanishing solution.

The Fresnel equation describes the wave surface. In our case solving the algebraic equation (A22) at $q_{x}=n_{m} \sin \theta_{\text {inc }}$ gives the values of the $z$ component of the vector $\mathbf{q}, q_{z}$. Generally, there are four roots of Eq. (A22), $\left\{q_{z}^{(1)}, q_{z}^{(2)}, q_{z}^{(3)}, q_{z}^{(4)}\right\}$, that form a set of the eigenvalues $\left\{\lambda_{1}, \lambda_{2}, \lambda_{3}, \lambda_{4}\right\}$ defined in Eq. (40). Each root $q_{z}^{(i)}$ corresponds to the eigenwave propagating inside the cell with the dimensionless wave vector $\mathbf{q}_{i}=\left(q_{x}, 0, q_{z}^{(i)}\right)$ and the refractive index $n_{i}=q_{i}$. The corresponding polarization vector of the electric displacement field is given by

$$
\mathbf{D}^{(i)}= \begin{cases}\cos \phi_{i} e_{1}\left(\hat{\mathbf{k}}_{i}\right)+\sin \phi_{i} e_{2}\left(\hat{\mathbf{k}}_{i}\right), & \left.c\right|_{q_{z}=q_{z}^{(i)}}>0, \\ -\sin \phi_{i} e_{1}\left(\hat{\mathbf{k}}_{i}\right)+\cos \phi_{i} e_{2}\left(\hat{\mathbf{k}}_{i}\right), & \left.c\right|_{q_{z}=q_{z}^{(i)}}<0,\end{cases}
$$

where

$$
\begin{aligned}
2 \phi_{i}=\left.\arg \left(a_{-}+i b\right)\right|_{q_{z}=q_{z}^{(i)}=} & \arg \left[\Delta \eta_{1}\left(\tilde{d}_{1}+i \tilde{d}_{2}\right)^{2}+\Delta \eta_{2}\left(\tilde{m}_{1}\right.\right. \\
& \left.\left.+i \tilde{m}_{2}\right)^{2}\right]_{q_{z}=q_{z}^{(i)}}
\end{aligned}
$$

From Eq. (A24) it is clear that the azimuthal angle $\phi_{i}$ becomes indeterminate in the degenerate case when the coefficients $a_{-}$and $b$ are both identically equal to zero. Typically, as far as the eigenmodes are concerned, this case does not present any fundamental difficulties. It just means that the azimuthal angles of the degenerate eigenmodes can be prescribed arbitrarily. Such freedom of choice, however, does not affect the evolution operator which remains uniquely defined.
The procedure to determine the characteristics of the eigenmodes involves the following steps: (a) evaluation of the eigenvalues $q_{z}^{(i)}$ by solving the Fresnel equation (A22); (b) calculation of the polarization vectors of the electric displacement field $\mathbf{D}^{(i)}$ by using the formula (A23); (c) computing the polarization vectors of the electric and magnetic fields from the relations: $\mathbf{E}^{(i)}=\mu \boldsymbol{\eta} \cdot \mathbf{D}^{(i)}[$ see Eq. (A15)] and $\mu \mathbf{H}^{(i)}=\mathbf{q}_{i} \times \mathbf{E}^{(i)}$ [see Eq. (16)].

As a result, we obtain the eigenvectors expressed as follows:

$$
\begin{aligned}
\mathbf{V}_{i} \propto\left(\begin{array}{c}
\mathbf{E}_{P}^{(i)} \\
\mathbf{H}_{P}^{(i)}
\end{array}\right), \quad \mathbf{H}_{P}^{(i)}=\left(\begin{array}{c}
q_{i} D_{1}^{(i)} \\
q_{z}^{(i)} D_{2}^{(i)}
\end{array}\right), \\
\mu^{-1} \mathbf{E}_{P}^{(i)}=\left(\begin{array}{c}
q_{z}^{(i)} D_{1}^{(i)} / q_{i} \\
D_{2}^{(i)}
\end{array}\right)+q_{x}\left[\Delta \eta_{1}\left(\mathbf{q}_{i}, \hat{\mathbf{d}}\right)\left(\hat{\mathbf{d}}, \mathbf{D}^{(i)}\right)\right. \\
\left.+\Delta \eta_{2}\left(\mathbf{q}_{i}, \hat{\mathbf{m}}\right)\left(\hat{\mathbf{m}}, \mathbf{D}^{(i)}\right)\right]\left(\begin{array}{l}
1 \\
0
\end{array}\right) .
\end{aligned}
$$

\section{Uniaxial anisotropy}

Now we apply the above procedure to the limiting case of uniaxial anisotropy with $\epsilon_{2}=\epsilon_{3}$ and $\Delta \epsilon_{2}=\Delta \eta_{2}=0$. At $\Delta \eta_{2}$ $=0$, the Fresnel equation (A22) takes the factorized form and the values of $q_{z}$ can be found as roots of two quadratic equations.

The first equation $1-\eta_{3} q^{2}=0$ represents the spherical wave surface. The corresponding eigenmodes are known as the ordinary waves. There are two values of $q_{z}$,

$$
q_{z}^{( \pm o)}= \pm \sqrt{n_{\perp}^{2}-q_{x}^{2}},
$$

where $n_{\perp}^{2}=\mu \epsilon_{3} \equiv \mu \epsilon_{\perp}$, that are equal in value but opposite in sign. When, similar to the incident and transmitted waves, the $z$ component of the wave vector (and the vector $\mathbf{q}$ ) is positive, the eigenmode might be called the refracted (forward) eigenwave. In the opposite case where, similar to the reflected wave, $q_{z}^{(\alpha)}$ is negative, the eigenmode will be referred to as the reflected (backward) eigenwave. So, Eq. (A27) describes two ordinary eigenmodes: the refracted eigenwave with $q_{z}=q_{z}^{(+o)}>0$ and the reflected eigenwave with $q_{z}=q_{z}^{(-o)}<0$.

The second equation

$$
q^{2}+u_{a}(\mathbf{q}, \hat{\mathbf{d}})^{2}-n_{\|}^{2}=0,
$$

where $n_{\|}^{2}=\mu \epsilon_{1} \equiv \mu \epsilon_{\|}$and $u_{a}=-\Delta \eta_{1} / \eta_{1}=\left(n_{\|}^{2}-n_{\perp}^{2}\right) / n_{\perp}^{2}$ is the anisotropy parameter, gives the values of $q_{z}$ for the eigenmodes known as the extraordinary waves. These are given by

$$
\begin{aligned}
& q_{z}^{( \pm e)}=\left[1+u_{a} d_{z}^{2}\right]^{-1}\left\{-u_{a} d_{z} d_{x} q_{x} \pm \sqrt{D}\right\}, \\
& D=n_{\|}^{2}\left(1+u_{a} d_{z}^{2}\right)-q_{x}^{2}\left[1+u_{a}\left(d_{x}^{2}+d_{z}^{2}\right)\right],
\end{aligned}
$$

where $d_{x}=(\hat{\mathbf{d}}, \hat{\mathbf{x}})$ and $d_{z}=(\hat{\mathbf{d}}, \hat{\mathbf{z}})$.

At $u_{a}>0\left(u_{a}<0\right)$, in the $x-z$ plane, Eq. (A28) describes the ellipse with the major (minor) semiaxis of the length $n_{\|}$ 
oriented perpendicular to the projection of the director (4) on the plane of incidence $\left(d_{x}, 0, d_{z}\right)$. The length of minor (major) semiaxis, $\tilde{n}_{\perp}=\left[n_{\perp}^{-2}-u_{a}\left(d_{y} / n_{\|}\right)^{2}\right]^{-1 / 2}$, depends on the $y$ component of the director and varies from $n_{\perp}$ to $n_{\|}$as $d_{y}^{2}$ increases from zero to unity. Clearly, degeneracy in refractive indices with $n_{o}=n_{ \pm e}$ may occur only if the director is in the incidence plane $\left(\phi_{d}=0\right)$. Additionally, the matching condition for the $x$ components of $\mathbf{q}$ and the director $q_{x} \equiv n_{m} \sin \theta_{\text {inc }}$ $= \pm n_{o} d_{x} \equiv \pm n_{o} \sin \theta_{d}$ needs to be met.

The wave vectors and the refractive indices of the normal modes are determined by the relation

$$
\mathbf{q}_{ \pm \alpha}=k_{\mathrm{vac}}^{-1} \mathbf{k}_{ \pm \alpha}=q_{x} \hat{\mathbf{x}}+q_{z}^{( \pm \alpha)} \hat{\mathbf{z}}=n_{ \pm \alpha} \hat{\mathbf{k}}_{ \pm \alpha}, \quad \alpha \in\{o, e\},
$$

where $n_{ \pm \alpha}=q_{ \pm \alpha}, n_{ \pm o}=n_{o}=n_{\perp}$ is the ordinary refractive index and $n_{ \pm e}$ is the refractive index of the extraordinary wave propagating along the unit vector $\hat{\mathbf{k}}_{ \pm e}$.

By substituting $\Delta \eta_{2}=0$ into Eq. (A23) we can obtain the polarization vectors of the electric displacement field $[29,30]$ as follows:

$$
\begin{gathered}
\mathbf{D}^{( \pm o)}=-q_{ \pm o}^{2} \mathbf{q}_{ \pm o} \times \hat{\mathbf{d}}=-n_{\perp}^{2} \mathbf{q}_{ \pm o} \times \hat{\mathbf{d}}, \\
\mathbf{D}^{( \pm e)}=q_{ \pm e}^{2} \mathbf{P}\left(\hat{\mathbf{k}}_{ \pm e}\right) \cdot \hat{\mathbf{d}}=q_{ \pm e}^{2} \hat{\mathbf{d}}-\left(\hat{\mathbf{d}}, \mathbf{q}_{ \pm e}\right) \mathbf{q}_{ \pm e} .
\end{gathered}
$$

Then, following the procedure described at the end of the preceding section, we find the polarization vectors of the electric field for the eigenmodes

$$
\begin{gathered}
\mu^{-1} \mathbf{E}^{( \pm o)}=-\mathbf{q}_{ \pm o} \times \hat{\mathbf{d}}, \\
\mu^{-1} \mathbf{E}^{( \pm e)}=\hat{\mathbf{d}}-n_{\perp}^{-2}\left(\hat{\mathbf{d}}, \mathbf{q}_{ \pm e}\right) \mathbf{q}_{ \pm e} .
\end{gathered}
$$

The result for the magnetic field of the normal modes is

$$
\begin{gathered}
\mathbf{H}^{( \pm o)}=n_{\perp}^{2} \hat{\mathbf{d}}-\left(\hat{\mathbf{d}}, \mathbf{q}_{ \pm o}\right) \mathbf{q}_{ \pm o}, \\
\mathbf{H}^{( \pm e)}=\mathbf{q}_{ \pm e} \times \hat{\mathbf{d}} .
\end{gathered}
$$

Solution of the eigenvalue problem can now be presented in the matrix form as follows:

$$
\Lambda=\operatorname{diag}\left(q_{z}^{(+e)}, q_{z}^{(+o)}, q_{z}^{(-e)}, q_{z}^{(-o)}\right), \quad \mathbf{V}=\left(\begin{array}{cc}
\mathbf{E}_{+} & \mathbf{E}_{-} \\
\mathbf{H}_{+} & \mathbf{H}_{-}
\end{array}\right)
$$

where

$$
\mathbf{E}_{ \pm}=\left(\begin{array}{cc}
E_{x}^{( \pm e)} & E_{x}^{( \pm o)} \\
E_{y}^{( \pm e)} & E_{y}^{( \pm o)}
\end{array}\right), \quad \mathbf{H}_{ \pm}=\left(\begin{array}{cc}
H_{y}^{( \pm e)} & H_{y}^{( \pm o)} \\
-H_{x}^{( \pm e)} & -H_{x}^{( \pm o)}
\end{array}\right) .
$$

Owing to the orthogonality relations (A6) the matrix

$$
\mathbf{N}=\mathbf{V}^{T} \cdot \mathbf{G} \cdot \mathbf{V}=\operatorname{diag}\left(\mathbf{N}_{+}, \mathbf{N}_{-}\right)
$$

is diagonal and its nonvanishing elements

$$
\mathbf{N}_{ \pm}=\left(\begin{array}{cc}
N_{ \pm e} & 0 \\
0 & N_{ \pm o}
\end{array}\right), \quad N_{\alpha}=2\left(\hat{\mathbf{z}}, \mathbf{E}^{(\alpha)} \times \mathbf{H}^{(\alpha)}\right)=2\left(\mathbf{E}_{P}^{(\alpha)}, \mathbf{H}_{P}^{(\alpha)}\right)
$$

are proportional to the normal components of the Poynting vector of the eigenmodes.

Substituting the expressions for the electric and magnetic fields of the eigenmodes [see Eqs. (A33) and (A34), respectively] into Eq. (A38) gives the diagonal elements of the matrices $\mathbf{N}_{+}$and $\mathbf{N}_{-}$,

$$
\begin{gathered}
\mu^{-1} N_{ \pm o}=2 q_{z}^{( \pm o)}\left[n_{\perp}^{2}-\left(\mathbf{q}_{ \pm o}, \hat{\mathbf{d}}\right)^{2}\right], \\
\mu^{-1} N_{ \pm e}=2 n_{\perp}^{-2}\left\{q_{z}^{( \pm e)}\left[n_{\perp}^{2}-\left(\mathbf{q}_{ \pm e}, \hat{\mathbf{d}}\right)^{2}\right]+d_{z}\left(\mathbf{q}_{ \pm e}, \hat{\mathbf{d}}\right)\left[n_{ \pm e}^{2}-n_{\perp}^{2}\right]\right\} .
\end{gathered}
$$

The eigenvalues of ordinary and extraordinary waves that enter the matrix of eigenvalues, $\boldsymbol{\Lambda}$, are given by Eqs. (A27) and (A29), respectively. The elements of the $2 \times 2$ block matrices (A36) that define the matrix of eigenvectors (A35) are the lateral components of the vectors (A33) and (A34).

These analytical expressions can now be substituted into the linking matrix (A8) to write explicitly the elements of the transfer matrix (36). For the homeotropic structure with $\hat{\mathbf{d}}$ $=\hat{\mathbf{z}}$, it is rather straightforward to show that the result is given by Eqs. (41)-(46).
[1] P. M. Chaikin and T. C. Lubensky, Principles of Condensed Matter Physics (Cambridge University Press, Cambridge, 1995)

[2] N. D. Mermin, Rev. Mod. Phys. 51, 591 (1979).

[3] L. Michel, Rev. Mod. Phys. 52, 617 (1980).

[4] H.-R. Trebin, Adv. Phys. 31, 195 (1982).

[5] M. Kléman, Points, Lines and Walls in Liquid Crystals, Magnetic Systems and Various Ordered Media (Wiley, NY, 1984).

[6] M. Kleman and J. Friedel, Rev. Mod. Phys. 80, 61 (2008).

[7] M. M. Salomaa and G. E. Volovik, Rev. Mod. Phys. 59, 533 (1987).

[8] M. Monastyrsky, Topology of Gauge Fields and Condensed
Matter (Plenum Press, NY, 1993).

[9] A. S. Schwarz, Quantum Field Theory and Topology (Springer, Berlin, 1993).

[10] J. F. Nye, Proc. R. Soc. London, Ser. A 389, 279 (1983).

[11] J. F. Nye and J. V. Hajnal, Proc. R. Soc. London, Ser. A 409, 21 (1987).

[12] J. F. Nye, Natural Focusing and Fine Structure of Light: Caustics and Wave Dislocations (Institute of Physics Publishing, Bristol, 1999).

[13] J. V. Hajnal, Proc. R. Soc. London, Ser. A 414, 433 (1987).

[14] J. V. Hajnal, Proc. R. Soc. London, Ser. A 414, 447 (1987).

[15] J. V. Hajnal, Proc. R. Soc. London, Ser. A 430, 413 (1990). 
[16] I. Freund and N. Shvartsman, Phys. Rev. A 50, 5164 (1994).

[17] I. Freund, A. I. Mokhun, M. S. Soskin, O. V. Angelsky, and I. I. Mokhun, Opt. Lett. 27, 545 (2002).

[18] M. V. Berry and M. R. Dennis, Proc. R. Soc. London, Ser. A 456, 2059 (2000).

[19] M. V. Berry and M. R. Dennis, Proc. R. Soc. London, Ser. A 457, 141 (2001).

[20] A. I. Konukhov and A. L. Melnikov, J. Opt. B: Quantum Semiclassical Opt. 3, S139 (2001).

[21] I. Freund, Opt. Commun. 201, 251 (2002).

[22] I. Freund, M. S. Soskin, and A. I. Mokhun, Opt. Commun. 208, 223 (2002).

[23] A. I. Mokhun, M. S. Soskin, and I. Freund, Opt. Lett. 27, 995 (2002).

[24] M. R. Dennis, Opt. Commun. 213, 201 (2002).

[25] M. V. Berry and M. R. Dennis, Proc. R. Soc. London, Ser. A 459, 1261 (2003).

[26] M. V. Berry, Proc. R. Soc. London, Ser. A 461, 2071 (2005).

[27] F. Flossmann, U. T. Schwarz, M. Maier, and M. R. Dennis, Phys. Rev. Lett. 95, 253901 (2005).

[28] F. Flossmann, U. T. Schwarz, M. Maier, and M. R. Dennis, Opt. Express 14, 11402 (2006).

[29] A. D. Kiselev, e-print arXiv:physics/0612114.

[30] A. D. Kiselev, J. Phys.: Condens. Matter 19, 246102 (2007).

[31] P. G. de Gennes and J. Prost, The Physics of Liquid Crystals (Clarendon Press, Oxford, 1993).

[32] P. Yeh and C. Gu, Optics of Liquid Crystal Displays (Wiley, Singapore, 1999).

[33] V. G. Chigrinov, Liquid Crystal Devices: Physics and Applications (Artech House, Boston, 1999).

[34] L. J. Yu and A. Saupe, Phys. Rev. Lett. 45, 1000 (1980).

[35] L. A. Madsen, T. J. Dingemans, M. Nakata, and E. T. Samulski, Phys. Rev. Lett. 92, 145505 (2004).

[36] L. Komitov, G. Hauk, and H. D. Koswig, Cryst. Res. Technol. 19, 253 (1984).

[37] L. H. Brett and H. H. Winter, Appl. Opt. 40, 2089 (2001).

[38] E. Gorecka, A. D. L. Chandani, Y. Ouchi, H. Takezoe, and A. Fukuda, Jpn. J. Appl. Phys., Part 1 29, 131 (1990).

[39] L. Parry-Jones, E. Kriezis, and S. Elston, Jpn. J. Appl. Phys.,
Part 2 41, L1485 (2002).

[40] S. Suwa, H. Hoshi, Y. Takanishi, K. Ishikawa, H. Takezoe, and B. Zeks, Jpn. J. Appl. Phys., Part 1 42, 1335 (2003).

[41] M. S. Soskin, V. Denisenko, and I. Freund, Opt. Lett. 28, 1475 (2003).

[42] M. S. Soskin, V. Denisenko, and R. Egorov, J. Opt. A, Pure Appl. Opt. 6, S281 (2004).

[43] R. G. Vovk, A. D. Kiselev, I. A. Buinyi, and M. S. Soskin, Ukr. J. Phys. 52, 748 (2007).

[44] M. Born and E. Wolf, Principles of Optics, 2nd ed. (Pergamon Press, Oxford, 1980).

[45] G. R. Luckhurst, Thin Solid Films 393, 40 (2001).

[46] G. R. Luckhurst, Nature (London) 430, 413 (2004).

[47] K. Severing and K. Saalwächter, Phys. Rev. Lett. 92, 125501 (2004).

[48] D. W. Berreman, J. Opt. Soc. Am. 62, 502 (1972).

[49] C. Oldano, Phys. Rev. A 40, 6014 (1989).

[50] D. A. Yakovlev, Opt. Spectrosc. 84, 923 (1998).

[51] M. Born and E. Wolf, Principles of Optics: Electromagnetic Theory of Propagation, Interference and Diffraction of Light, 7th ed. (Cambridge University Press, New York, 1999).

[52] Ellipsometry and Polarized Light, edited by R. M. A. Azzam and N. M. Bashara (North Holland Publishing Company, Amsterdam, 1977).

[53] J. D. Jackson, Classical Electrodynamics, 3rd ed. (Wiley, New York, 1999).

[54] M. V. Berry, J. Opt. A, Pure Appl. Opt. 6, 675 (2004).

[55] M. V. Berry and J. H. Hannay, J. Phys. A 10, 1809 (1977).

[56] P. C. Hohenberg and B. I. Halperin, Rev. Mod. Phys. 49, 435 (1977).

[57] R. L. Devaney, An Introduction to Chaotic Dynamical Systems, 2nd ed. (Addison-Wesley, Sydney, 1989).

[58] B. A. Dubrovin, A. T. Fomenko, and S. P. Novikov, Modern Geometry-Methods and Applications, Springer Series in Soviet Mathematics Vol. 3 (Springer, NY, 1990).

[59] M. V. Berry and M. R. Dennis, J. Phys. A: Math. Theor. 40, 65 (2007).

[60] A. Yariv and P. Yeh, Optical Waves in Crystals: Propagation and Control of Laser Radiation (Wiley, New York, 1984). 